\title{
Lyndon factorization of generalized words of Thue
}

\author{
Anton Černý ${ }^{\dagger}$ \\ Department of Mathematics and Computer Science, Kuwait University, P.O. Box 5969 Safat 13060, Kuwait
}

received Jul 17, 2000, revised Jul, 2001, accepted Mar 24, 2002.

The $i$-th symbol of the well-known infinite word of Thue on the alphabet $\{0,1\}$ can be characterized as the parity of the number of occurrences of the digit 1 in the binary representation of $i$. Generalized words of Thue are based on counting the parity of occurrences of an arbitrary word $w \in\{0,1\}^{*}-0^{*}$ in the binary representation of $i$. We provide here the standard Lyndon factorization of some subclasses of this class of infinite words.

Keywords: Lyndon word, Lyndon factorization, automatic sequence

\section{Introduction}

When we are interested in getting a better insight into the structure of some object, whether in mathematics or in computer science, one of the basic approaches how to tackle the problem is decomposition of the object into smaller "canonical" objects. The classical example is the factorization of a natural number into prime numbers being a powerful tool in the number theory. Natural numbers coded in unary notation correspond to finite words (strings) over a single-letter alphabet. Words over arbitrary alphabets can be considered to be a generalization of the concept of the natural number. A canonical decomposition of words to concatenation factors being Lyndon words is provided by the Lyndon factorization theorem ([10]). Lyndon words are primitive words minimal in their conjugacy classes. They are easy to deal with, e.g., there exists a simple linear algorithm, introduced by Duval ([8], [2]) for generating Lyndon words, up to a given length, in lexicographic order. A structural relationship of Lyndon words to other important classes of words has been established ([U]]). Application of the Lyndon factorization leads to important results in the investigation of finite factorizations of the free monoid $A^{*}$ and to discovery of new unavoidable regularities in words ([20]). Similar investigations of factorizations were performed for the case of free partially commutative monoids ([]]). The Lyndon factorization can be generalized to Viennot factorization by proceeding from the lexicographic ordering to Viennot orderings ([13]).

The Lyndon-factorization theorem has been further extended to infinite words (infinite sequences of symbols - [117]). Investigation of infinite words is crucial in the area of combinatorics on words, since properties of infinite words very often imply regularities in infinite sets of finite words. It is quite natural

\footnotetext{
${ }^{\dagger}$ This work was supported by the Kuwait University grant SM-178 
that, after the existence of the unique Lyndon factorization of infinite words had been proved, the factorization of some important sequences was investigated. In particular, the factorization of the sequence of Thue, of the Fibonacci sequence, of the regular paperfolding sequence and of the characteristic Sturmian sequences was characterized in [9], [11] ], [12].

The sequence of Thue was historically the first sequence where the structural properties were systematically studied ([18], [19]; a construction of that sequence was mentioned, probably for the first time, in [14]). The Thue sequence is one of the simplest non-periodic sequences in the important class of automatic sequences (originally called uniform tag sequences - [6]). Automatic sequences can be equivalently characterized by, among others, finite state automata, uniform morphisms or substitutions ([6], [5]).

In the current paper we investigate the Lyndon factorization of several subclasses of the class of generalized sequences of Thue. The generalized Thue sequences were first introduced in [5]. Each word $w$ on the alphabet $\{0,1\}$, which contains at least one symbol 1 , describes, as a parameter, a sequence $t_{w}$ in this class. The $i$-th symbol of $t_{w}$ corresponds to the parity of occurrences of $w$ as a factor of the binary representation for $i$. The Thue sequence corresponds to the single-letter parameter word 1. As is well known, the Thue sequence does not contain cubic factors. Similarly, the sequence $t_{w}$ does not contain a factor, which is the $2^{|w|}$-th power of a non-empty word ([[3]). In fact, $t_{w}$ does not contain any cubic factors except powers of a single letter ([15],[16]).

The present work is, in a sense, of an experimental character. We believe that studying the factorization pattern of a whole class of structurally related infinite sequences may bring some deeper insight to the relationship between the structure of a sequence and its Lyndon decomposition. Though we provide here a description of the Lyndon factorization of just a few subclasses of the generalized Thue sequences, it seems to be apparent that the Lyndon factors follow the block structure of the sequence determined by the iteration underlying uniform morphism or substitution. On the other hand, there does not seem to be a straightforward way leading to the unique description of the factorization of the whole class of the generalized words of Thue.

\section{Notation and basic notions}

\subsection{Preliminaries}

We denote as $\mathbb{N}=\{0,1, \ldots\}$ the set of all natural numbers and, for $p \in \mathbb{N}$, we denote $[p]=\{i \in \mathbb{N} ; i<p\}$. An alphabet is any finite non-empty set $\Sigma$ (its elements are called symbols). In particular, we will use the set $[2]=\{0,1\}$ as an alphabet consisting of 2 symbols. A finite word $w$ of length $|w|=n \in \mathbb{N}$ on $\Sigma$ is a finite sequence $w=a_{0} a_{1} \cdots a_{n-1}$ of symbols from $\Sigma$. The empty word (of length 0 ) is denoted by $\lambda$. An infinite word (a sequence for short) on $\Sigma$ is an infinite sequence $w=a_{0} a_{1} \cdots$ of symbols from $\Sigma$. The symbol in a word or sequence $w$ at the position $i$ is denoted as $w(i)$ (the position numbering starts from 0$)$. By $\Sigma^{*}, \Sigma^{p},\left(\Sigma^{p}\right)^{*}, \Sigma^{\omega}$ we denote the sets of all words, of all words of length $p$, of all words of length being a multiple of $p$ and of all sequences on $\Sigma$, respectively. Further we denote $\Sigma^{\infty}=\Sigma^{*} \cup \Sigma^{\omega}$ and $\left(\Sigma^{p}\right)^{\infty}=\left(\Sigma^{p}\right)^{*} \cup \Sigma^{\omega}$.

For a number $i \in\left[2^{k}\right]$ we denote as $i_{[2], k} \in[2]^{k}$ the word being the binary representation of $i$ of length $k \geq 0$. For a word $w \in[2]^{k}$ we denote as $[w]_{2}$ the unique number from $\left[2^{k}\right]$ with binary representation $w$.

The concatenation of the words $x \in \Sigma^{*}$ and $y \in \Sigma^{\infty}$ is $x y \in \Sigma^{\infty}$. For $x, y \in \Sigma^{*}$, the words $x y$ and $y x$ are called conjugates. If $w=x y z \in \Sigma^{\infty}$, then $x, y, z$ are called a prefix, a factor at position $|x|$, and a suffix of $w$, respectively. In this case, we further denote $x^{-1} w=y z$ and $w z^{-1}=x y$. A prefix, factor, or suffix is called 
proper if the remaining part of $w$ is not empty. A word is primitive if it is not a concatenation of two or more equal factors. If $\left(w_{i}\right)_{i=0}^{\infty}$ is a sequence of finite words of unlimited length such that, for each $i \geq 0$, $w_{i}$ is a prefix $w_{i+1}$, then $\lim _{i \rightarrow \infty} w_{i}$ will denote the unique infinite word having each $w_{i}$ as a prefix.

Let $r \geq 1, s \geq 0$ and let $\left(\Sigma^{r}\right)^{*}=\bigcup_{k \in \mathbb{N}} \Sigma^{k r}$. A $(r, s)$-substitution (introduced in [5]) is a mapping $\mu$ : $\left(\Sigma^{r}\right)^{\infty} \rightarrow \Gamma^{\infty}$, satisfying $\mu\left(\Sigma^{r}\right) \subset \Gamma^{s}$, and $\mu(x y)=\mu(x) \mu(y)$ for $x \in\left(\Sigma^{r}\right)^{*}, y \in\left(\Sigma^{r}\right)^{\infty}$. Thus $\mu$ is completely determined by its values on $\Sigma^{r}$. The substitution $\mu$ is prolongable in a word $u \in \Sigma^{r}$ if $u$ is a proper prefix of $\mu(u)$. Then, clearly, $s>r \geq 1$ and the sequence $\left(\mu^{i}(u)\right)_{i=0}^{\infty}$ of words obtained by repeated application of $\mu$ starting from $u$ yields a limit $s=\lim _{i \rightarrow \infty} \mu^{i}(u)$ being a fixed point of $\mu$, i.e., satisfying $\mu(s)=s$. A $(1, p)$-substitution is called $p$-uniform morphism.

Automatic sequences are described by finite state automata ([6], where these sequences are called uniform tag sequences). We will limit here our considerations to 2-automatic sequences on the alphabet [2] only. A 2-finite-state automaton $(2-f s a)$ is a tuple $\mathfrak{A}=\left(\Sigma,[2], \delta, a_{0}, F\right)$ where $\Sigma$ is a (state) alphabet, $\delta$ : $\Sigma \times[2] \rightarrow \Sigma$ is the transition function, $a_{0} \in \Sigma$ is the initial state and $F \subset \Sigma$. We will assume that $\mathfrak{A}$ satisfies $\delta\left(a_{0}, 0\right)=a_{0}$. We will consider the usual extension $\delta^{*}: \Sigma \times[2]^{*} \rightarrow \Sigma$ defined for $a \in \Sigma, w \in[2]^{*}, x \in[2]$ inductively as $\delta^{*}(a, \lambda)=a, \delta^{*}(a, w x)=\delta\left(\delta^{*}(a, w), x\right)$. A sequence $s=b_{0} b_{1} \cdots \in[2]^{\omega}$ is 2-automatic if there exists a 2 -fsa $\mathfrak{A}$ as above such that, for $i \in \mathbb{N}, b_{i}=1$ iff $\delta^{*}\left(a_{0}, i_{[2],[\log (i+1)]}\right) \in F$. We say that $s$ is

described by $\mathfrak{A}$. We will use here several equivalent characterizations of 2 -automatic sequences - see [6], [4] for the proof of Theorem 2.1.1. (Remark 2.1.2 is based on this proof.)

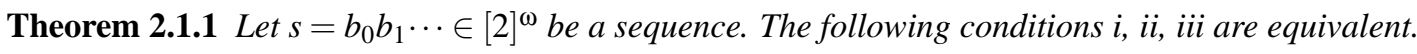

(i) $s$ is a 2-automatic sequence

(ii) there is an alphabet $\Sigma$, a 2-uniform morphism $\varphi: \Sigma^{\infty} \rightarrow \Sigma^{\infty}$ and a 1-uniform morphism $\psi: \Sigma^{\infty} \rightarrow[2]^{\infty}$ such that $s=\psi\left(\lim _{n \rightarrow \infty} \varphi^{n}(a)\right)$

(iii) $s$ is a fixed point of some $\left(k, k p^{m}\right)$-substitution $\mu:[2]^{\infty} \rightarrow[2]^{\infty}, k, m \geq 1$.

Remark 2.1.2 Let a 2-automatic sequence be described by the fsa $\mathfrak{A}=\left(\Sigma,[2], \delta, a_{0}, F\right)$. The morphisms $\varphi, \psi$ from 园 of Theorem 2.1.1 are given, for $a \in \Sigma$, as $\varphi(a)=\delta(a, 0) \delta(a, 1), \psi(a)=($ if $a \in F$ then 1 else $0)$. The symbol of $\psi\left(\varphi^{r}(a)\right)$ at position $i \in\left[2^{r}\right], r \geq 0$, is then $\psi\left(\delta^{*}\left(a, i_{[2], r}\right)\right)$.

\subsection{Lyndon words}

Assume a totally ordered alphabet $\Sigma$ with the corresponding lexicographic order on $\Sigma^{\infty}$. Lyndon words are defined as primitive words, which are minimal in the class of all their conjugates. The proof of the following equivalent characterization of Lyndon words and the proof of the Lyndon's theorem can be found in [10].

Proposition 2.2.1 A non-empty word is a Lyndon word iff it is strictly smaller than any of its non-empty proper suffixes.

Theorem 2.2.2 (Lyndon) Any non-empty finite word can be written uniquely as a concatenation of a non-increasing sequence of Lyndon words.

In [17] the authors defined infinite Lyndon words as those sequences, which have infinitely many prefixes being Lyndon words. They proved the following generalization of the Lyndon theorem. 
Theorem 2.2.3 Any infinite word s can be uniquely factorized in one of the following forms:

$$
\begin{array}{ll}
s=l_{0} l_{1} \cdots, & \text { where } l_{0} \geq l_{1} \geq \cdots \text { are finite Lyndon words } \\
\text { or } & \begin{array}{ll}
\text { where } l_{0} \geq l_{1} \geq \cdots \geq l_{k} \text { are finite Lyndon words } \\
s=l_{0} l_{1} \cdots l_{k} l_{k+1} & \text { and } l_{k+1}<l_{k} \text { is an infinite Lyndon word. }
\end{array}
\end{array}
$$

\subsection{Generalized words of Thue}

The infinite word of Thue $t$ can be defined as $t=\lim _{i \rightarrow \infty} \varphi^{i}(0)$ where $\varphi$ is the 2-uniform morphism on the alphabet $\{0,1\}$ described as $\varphi(0)=01, \varphi(1)=10$. Hence $t=0110100110010110 \cdots$. The digit at the $i$-th position of $t$ can be determined as the parity of the number of occurrences of the digit 1 in the binary representation of $i$. In [5] generalized words of Thue were introduced. They were further investigated in [3], [15] and [16]. Each such infinite word $t_{w}=t_{w}(0) t_{w}(1) t_{w}(2) \cdots \in\{0,1\}^{\omega}$ is based on a word $w \in\{0,1\}^{*}-\{0\}^{*}$. The symbol $t_{w}(i)$ at the $i$-th position of $t_{w}$ is defined as $t_{w}(i)=\#_{w} 0^{|w|} i_{[2]} \bmod 2$ where $\#_{w} 0^{|w|} i_{[2]}$ denotes the number of factors $w$ occurring in the binary representation of $i$ padded with at least $|w|$ leading zeroes. Every occurrence of a factor is counted, e.g., $t_{010}(10)=0$, since 0001010 (the binary representation of 10 padded with 3 leading zeroes) contains two overlapping factors 010 . In particular, $t=t_{1}$.

Let for the remaining part of the paper $w \in\{0,1\}^{*}-\{0\}^{*}$ be an arbitrary but fixed word. Denote $k=|w|, \Sigma_{w}=\{\langle\alpha, m\rangle ; \alpha$ is a proper prefix of $w, m \in[2]\}$. We will suppose that $k \geq 2$ since the case $k=1$ means $t_{w}$ is the sequence of Thue. For the purpose of the next theorem (the proof of the theorem can be found in [3] ), for each $x, y \neq \lambda$, let $\operatorname{sp}(x, y)$ denote the longest suffix of $x$ that is a proper prefix of $y$.

Theorem 2.3.1 The sequence $t_{w}$ is described by the $f_{s} a \mathfrak{A}_{w}=\left(\Sigma_{w},[2], \delta_{w}, a_{0, w}, F_{w}\right)$ where, for $\langle\alpha, m\rangle \in$ $\Sigma_{w}, j \in[2]$,

$$
\delta_{w}(\langle\alpha, m\rangle, j)=\langle s p(\alpha j, w), \text { if } \alpha j=w \text { then } 1-m \text { else } m\rangle,
$$

$a_{0, w}=\left\langle s p\left(0^{k}, w\right), 0\right\rangle$ and $F_{w}=\left\{\langle\alpha, 1\rangle ;\langle\alpha, 1\rangle \in \Sigma_{w}\right\}$. Moreover, $\mathfrak{A}_{w}$ is the minimal fsa, with respect to the size of the state alphabet, describing $t_{w}$.

We will denote as $\varphi_{w}, \psi_{w}$ the morphisms corresponding to $\mathfrak{A}_{w}$ according to Remark 2.1.2 (it is worth to observe that $\left.\psi_{w}(\langle\alpha, m\rangle)=m\right)$. The sequence $t_{w}$ is a fixed point of a substitution described by the following theorem from [5].

Theorem 2.3.2 Let $\mu_{w}$ be the $\left(2^{k-1}, 2^{k}\right)$-substitution defined on $[2]^{k-1}$ as

$$
\mu_{w}\left(x_{0} x_{1} \cdots x_{2^{k-1}-1}\right)=y_{0} y_{1} \cdots y_{2^{k}-1}
$$

where, for $i \in\left[2^{k}\right], y_{i}=\left(x_{\lfloor i / 2\rfloor}+\chi_{w}(i)\right) \bmod 2$, and $\chi_{w}(i)=$ if $w=i_{[2], k}$ then 1 else 0 . Then $\mu_{w}\left(t_{w}\right)=t_{w}$.

Property 2.3.3 For $i \geq 0$ and $a \in \Sigma_{w}, \mu_{w}^{i}\left(\psi_{w}\left(\varphi_{w}^{k-1}(a)\right)\right)=\psi_{w}\left(\varphi_{w}^{k-1+i}(a)\right)$.

Proof. Let $a \in \Sigma_{w}$. Since the automaton $\mathfrak{A}_{w}$ is minimal, the state $a$ can be reached from the initial state $a_{0, w}$. Remark 2.1.2 then implies that $a$ occurs in the sequence $\lim _{n \rightarrow \infty} \varphi^{n}\left(a_{0, w}\right)$. Let it be at position $j$. 
Then Theorem 2.3.2 implies that

$$
\begin{aligned}
\mu_{w}\left(\psi_{w}\left(\varphi_{w}^{k-1}(a)\right)\right) & =\mu_{w}\left(t_{w}\left(2^{k-1} j\right) \cdots\right. \\
\left.t_{w}\left(2^{k-1} j+2^{k-1}-1\right)\right) & =t_{w}\left(2^{k} j\right) \cdots \\
t_{w}\left(2^{k} j+2^{k}-1\right) & =\psi_{w}\left(\varphi_{w}^{k}(a)\right) \\
& =\psi w\left(\varphi_{w}^{k-1}(\varphi(a))\right) .
\end{aligned}
$$

The required assertion is obtained by a simple inductive argument.

Example 2.3.4 Let $w=010$. The sequence (spaces are inserted for better readability)

$$
t_{010}=001011001101000011010011001000001101 \cdots
$$

is described by the $f$ sa $\mathfrak{A}_{010}=\left(\Sigma_{010},[2], \delta_{010},\langle 0,0\rangle, F_{010}\right)$ where

$$
\begin{aligned}
\Sigma_{010} & =\{\langle\lambda, 0\rangle,\langle 0,0\rangle,\langle 01,0\rangle,\langle\lambda, 1\rangle,\langle 0,1\rangle,\langle 01,1\rangle\}, \\
F_{010} & =\{\langle\lambda, 1\rangle,\langle 0,1\rangle,\langle 01,1\rangle\}
\end{aligned}
$$

and the transition function is given by the following table

\begin{tabular}{||l||l|l||}
\hline \hline$\delta_{010}$ & 0 & 1 \\
\hline \hline$\langle\lambda, 0\rangle$ & $\langle 0,0\rangle$ & $\langle\lambda, 0\rangle$ \\
\hline$\langle 0,0\rangle$ & $\langle 0,0\rangle$ & $\langle 01,0\rangle$ \\
\hline$\langle 01,0\rangle$ & $\langle 0,1\rangle$ & $\langle\lambda, 0\rangle$ \\
\hline \hline
\end{tabular}

\begin{tabular}{||l||l|l||}
\hline \hline$\delta_{010}$ & 0 & 1 \\
\hline \hline$\langle\lambda, 1\rangle$ & $\langle 0,1\rangle$ & $\langle\lambda, 1\rangle$ \\
\hline$\langle 0,1\rangle$ & $\langle 0,1\rangle$ & $\langle 01,1\rangle$ \\
\hline$\langle 01,1\rangle$ & $\langle 0,0\rangle$ & $\langle\lambda, 1\rangle$ \\
\hline \hline
\end{tabular}

The morphism $\varphi_{010}$ is described by the rows of the table, e.g.,

$$
\varphi_{010}(\langle 0,1\rangle)=\langle 0,1\rangle\langle 01,1\rangle
$$

and

$$
\psi_{w}(\langle\alpha, m\rangle)=m \quad \text { for } \quad \alpha \in\{\lambda, 0,01\}, m \in\{0,1\} .
$$

The sequence $t_{010}$ is a fixed point of the substitution

$$
\begin{array}{rlrl}
0000 & \mapsto 00100000 & 1111 & \mapsto 11011111 \\
0010 & \mapsto 00101100 & 1101 & \mapsto 11010011 \\
1100 & \mapsto 11010000 & 0011 & \mapsto 00101111
\end{array}
$$

We will further use the following two easy observations without explicitly referring to them.

Observation 2.3.5 Let $r \geq 1$. $x \in \Sigma_{w}^{\infty}$. No symbol from $\Sigma$ can occur in $\varphi_{w}^{r}(x)$ both in an even and in an odd position.

Observation 2.3.6 For each $x \in \Sigma_{w}^{\infty}$ let $\bar{x}$ denote the word obtained by replacing each symbol $\langle\alpha, m\rangle$ by $\langle\alpha, 1-m\rangle$. Similarly, for each word $x \in[2]^{\infty}$ let $\bar{x}$ denote the word obtained by replacing each symbol $m$ by $1-m$. Then 
1. For each $a \in \Sigma_{w}$ and $r \geq 0, \varphi_{w}^{r}(\bar{a})=\overline{\varphi_{w}^{r}(a)}$

2. For each $a \in \Sigma_{w}$ and $r \geq 0, \psi_{w}\left(\varphi_{w}^{r}(\bar{a})\right)=\overline{\psi_{w}\left(\varphi_{w}^{r}(a)\right)}$

3. For each $x \in\left([2]^{k-1}\right)^{\infty}, \mu_{w}(\bar{x})=\overline{\mu_{w}(x)}$.

In the remaining part of this text we will omit the subscript $w$ from $\mathfrak{A}_{w}, \Sigma_{w}, \delta_{w}, \delta_{w}^{*}, a_{0, w}, F_{w}, \varphi_{w}, \psi_{w}, \mu_{w}$ and $\chi_{w}$.

\section{Generalized words of Thue and the lexicographic order}

\subsection{Lexicographic order on the set $\Sigma^{\infty}$}

We consider the set $[2]^{\infty}$ to be ordered lexicographically based on the ordering $0<1$ of [2]. We will investigate the factors of the sequence $t_{w}$ and their properties related to this lexicographic ordering. The structure of the sequence $t_{w}$ closely matches the structure of the sequence $\tau_{w}=\lim _{n \rightarrow \infty} \varphi^{n}\left(a_{0}\right) \in \Sigma^{\omega}$. It seems to be useful to look for an order relation on $\Sigma$ (and the corresponding lexicographic order on $\Sigma^{\infty}$ ) related to the order of the block factors of $\Sigma$ of the form $\psi\left(\varphi^{r}(a)\right)$, such that the morphism $\varphi$ is growing on $\Sigma^{\infty}$. Since $t_{w}$ is a fixed point of the $\left(2^{k-1}, 2^{k}\right)$-substitution $\mu$, one would expect that the relative order of two symbols $a, b \in \Sigma$ should be implied by the order of the words $\psi\left(\varphi^{k-1}(a)\right), \psi\left(\varphi^{k-1}(b)\right)$. This choice need not be (always) suitable as illustrated by the following example.

Example 3.1.1 Consider $w=10^{s}, s \geq 2$, hence $k-1=s, k-1+s=2 s$. Let $a=\langle 1,1\rangle, b=\left\langle 10^{s-1}, 1\right\rangle$. Then

$$
\begin{aligned}
& \psi\left(\delta^{*}\left(a, i_{[2], 2 s-1}\right)\right)=\left\{\begin{array}{ll}
0 & \text { for } i<2^{s-1} ; \\
1 & \text { for } i=2^{s-1} ;
\end{array} \quad \psi\left(\delta^{*}\left(a, i_{[2], 2 s}\right)\right)=0 \text { for } i \leq 2^{s} ;\right. \\
& \psi\left(\delta^{*}\left(b, i_{[2], 2 s-1}\right)\right)=0 \quad \text { for } i \leq 2^{s-1} ; \quad \psi\left(\delta^{*}\left(b, i_{[2], 2 s}\right)\right)= \begin{cases}0 & \text { for } i<2^{s} ; \\
1 & \text { for } i=2^{s} .\end{cases}
\end{aligned}
$$

Therefore $\psi\left(\varphi^{k-1+s-1}(a)\right)>\psi\left(\varphi^{k-1+s-1}(b)\right)$ and $\psi\left(\varphi^{k-1+s}(a)\right)<\psi\left(\varphi^{k-1+s}(b)\right)$.

For the rest of the paper, denote by $s$ the length of the longest suffix of $w$ belonging to $0^{*}$. Example 3.1.1 documented that choosing for the definition of the order relation on $\Sigma$ an exponent of $\varphi$ smaller than $k-1+s$ results in an order relation, with respect to which $\varphi$ is not a growing function. Therefore we will consider the ordering of $\Sigma$ based on the blocks $\psi\left(\varphi^{k-1+s}(a)\right)$.

Definition 3.1.2 The (total) order relation $<$ on $\Sigma$ is defined, for $a, b \in \Sigma$, as

$$
a<b \text { iff } \psi\left(\varphi^{k-1+s}(a)\right)<\psi\left(\varphi^{k-1+s}(b)\right) \text {. }
$$

The relation $<$ is indeed an order relation due to the following Lemma.

Lemma 3.1.3 Let $a, b \in \Sigma, a \neq b$. Then, for $r \geq 0, \psi\left(\varphi^{k-1+r}(a)\right) \neq \psi\left(\varphi^{k-1+r}(b)\right)$.

Proof. Let $a=\langle\alpha, m\rangle, b=\langle\beta, n\rangle, a \neq b$ and let $d \in[2]$ be different from the last symbol of $w$. We will use Remark 2.1.2. If $m=n$ then $\alpha \neq \beta$, assume $|\alpha|<|\beta|$. Let $w=\beta \gamma$. Then $\psi\left(\delta^{*}\left(a, \gamma d^{k-1+r-|\gamma|}\right)\right)=m$, $\psi\left(\delta^{*}\left(b, \gamma d^{k-1+r-\gamma \mid}\right)\right)=1-n \neq m$. If $m \neq n$ then $\psi\left(\delta^{*}\left(a, d^{k-1+r}\right)\right)=m \neq n=\psi\left(\delta^{*}\left(b, \gamma d^{k-1+r-|\gamma|}\right)\right)$. 


\subsection{Monotonicity of $\varphi$ and $\mu$}

The total order of $\Sigma$ induces the lexicographic order on $\Sigma^{\infty}$. We will denote $\Psi=\psi \circ \varphi^{k-1+s}$. Clearly, the $(k-1+s)$-uniform morphism $\Psi: \Sigma^{\infty} \rightarrow[2]^{\infty}$ is a strictly growing function. Property 2.1 .2 implies

Property 3.2.1 For $i \geq 0$ and $x \in \Sigma^{*}, \mu^{i}(\Psi(x))=\Psi\left(\varphi^{i}(x)\right)$.

We want to show that the morphism $\varphi$ is strictly growing, as well. To prove it we need Property 3.2.2, which was proved in [3] and one more technical lemma.

Property 3.2.2 $\varphi$ is a one-to-one function.

Lemma 3.2.3 If $a, b \in \Sigma, a \neq b$, and $r \geq 0$ is the first position where $\Psi(a)$ and $\Psi(b)$ contain a different symbol then $r$ is a multiple of $2^{s}$.

Proof. Assume $r$ is not a multiple of $2^{s}$, i.e., $r \geq 1$ and $0^{s}$ is not a suffix of $r_{[2], k-1+s}$. Denote $\rho$ the word obtained from $r_{[2], k-1+s}$ by removing the last symbol. Let $a=\langle\alpha, m\rangle, b=\langle\beta, n\rangle$. Since neither of $\alpha r_{[2], k-1+s}, \beta r_{[2], k-1+s}$ contains the suffix $w$, we have

$$
\begin{aligned}
& \psi\left(\delta^{*}\left(a, r_{[2], k-1+s}\right)\right)=\psi\left(\delta^{*}(a, \rho)\right) \text { and } \\
& \psi\left(\delta^{*}\left(b, r_{[2], k-1+s}\right)\right)=\psi\left(\delta^{*}(b, \rho)\right),
\end{aligned}
$$

hence $\psi\left(\varphi^{k-1+s}(a)\right)$ and $\psi\left(\varphi^{k-1+s}(b)\right)$ differ in position $\lfloor r / 2\rfloor<r$ - a contradiction to the minimality of $r$.

Lemma 3.2.4 For $a, b \in \Sigma$, if $a<b$ then $\varphi(a)<\varphi(b)$.

Proof. Let $a<b$ (i.e., $\Psi(a)<\Psi(b)$ ) and let $r$ be the first position in which $\Psi(a)$ and $\Psi(b)$ differ. Then $\Psi(a)(r)=0$ and $\Psi(b)(r)=1$. Assume by contrary that $\varphi(a) \geq \varphi(b)$, i.e., $\varphi(a)>\varphi(b)$ as implied by Property 3.2.2. Then $\Psi(\varphi(a))>\Psi(\varphi(b))$. Considering Proposition 3.2.1, this means $\mu(\Psi(a))>$ $\mu(\Psi(b))$. Theorem 2.3.2 implies that $\mu(\Psi(a))$ and $\mu(\Psi(b))$ have a common prefix of length $2 r$. Moreover, the "correction" caused by the function $\chi$ must take place at position $2 r$, since otherwise $\mu(\Psi(a))(2 r)=$ $\Psi(a)(r)<\Psi(b)(r)=\mu(\Psi(b))(2 r)$ and, consequently, $\mu(\Psi(a))<\mu(\Psi(b))$. Therefore $2 r=2^{k} t+[w]_{2}$ for some $t \geq 0$ and Lemma 3.2.3 implies that $[w]_{2}$ is a multiple of $2^{s+1}$ - a contradiction to the maximality of $s$.

Corollary 3.2.5 $\varphi$ is strictly growing on $\Sigma^{\infty}$.

The following Corollary 3.2.6 follows from Property 3.2.1.

Corollary 3.2.6 $\mu$ is strictly growing on $\left\{\Psi(x) ; x \in \Sigma^{\infty}\right\}$.

In the remaining part of the paper we will provide the characterization of the Lyndon factorization of the sequence $t_{w}$ for four different shapes of the word $w$.

\section{Factorization of four types of generalized words of Thue.}

We consider here four types of generalized Thue sequences, based on the shape of the underlying word $w$. For each type we provide a theorem describing the Lyndon factorization of the sequence $t_{w}$. Typically, the Lyndon factorization of $t_{w}$ starts by an initial finite sequence of Lyndon factors (we will call this part the "preamble" of the factorization), followed by a product of a recurrently described infinite sequence of Lyndon factors (the "body" of the factorization). The length of the preamble may grow with the increasing 
length of the word $w$. The recurrent description of the body is based on the generating substitution $\mu_{w}$. Proofs of the theorems from this section can be found in Section 1 .

Each theorem is followed by an example of factorization of one sequence $t_{w}$ of the particular type, together with the corresponding mappings $\mu_{w}$ and $\varphi_{w}$. In the examples, we include spaces to increase readability of $t_{w}$. The first symbol of each Lyndon factor of $t_{w}$ is underlined. The morphism $\varphi_{w}$ is given for arguments of the form $\langle\alpha, 0\rangle$ only. The values for arguments of the form $\langle\alpha, 1\rangle$ can be obtained by replacing each 0 or 1 in the second position by the complementary value.

\subsection{Case $w=0^{k-1} 1$}

Theorem 4.1.1 Let $w=0^{k-1} 1$. Then $t_{w}=\prod_{i=0}^{\infty} w_{i}$ where $w_{0}=01^{2^{k}}, w_{1}=01^{2^{k}-1}, w_{i+1}=1^{-1} \mu\left(1 w_{i} 1^{-1}\right) 1$ for $i>1$. For $i \geq 0$, the word $w_{i}$ is a Lyndon word and $w_{i}>w_{i+1}$.

Example 4.1.2 $w=001 \quad(k=3)$

\begin{tabular}{|c|c|c|}
\hline \multicolumn{3}{|l|}{$t_{001}$} \\
\hline \multicolumn{3}{|c|}{$\underline{0} 111111110111111100011111011111110000000101111111000111110111111$} \\
\hline \multicolumn{3}{|c|}{1000000001000000100011111011111110000000101111111000111110111111} \\
\hline \multicolumn{3}{|c|}{1000000001000000011100000100000010000000101111111000111110111111} \\
\hline \multicolumn{3}{|c|}{1000000001000000100011111011111110000000101111111000111110111111} \\
\hline \multicolumn{3}{|c|}{1000000001000000011100000100000001111111010000000111000001000000} \\
\hline \multicolumn{3}{|c|}{1000000001000000100011111011111110000000101111111000111110111111} \\
\hline \multicolumn{3}{|c|}{1000000001000000011100000100000010000000101111111000111110111111} \\
\hline \multirow{3}{*}{\multicolumn{3}{|c|}{$\begin{array}{l}1000000001000000100011111011111110000000101111111000111110111111 \\
1000000001000000011100000100000001111111010000000111000001000000 \\
01111111101111110111000001000000011111110100000001110000 \ldots\end{array}$}} \\
\hline & & \\
\hline & & \\
\hline$\mu_{001}$ & & $\varphi_{001}$ \\
\hline $0000 \mapsto 01000000$ & $1111 \mapsto 10111111$ & $\langle\lambda, 0\rangle \mapsto\langle 0,0\rangle\langle\lambda, 0\rangle$ \\
\hline $0100 \mapsto 01110000$ & $1011 \mapsto 10001111$ & $\langle 0,0\rangle \mapsto\langle 00,0\rangle\langle\lambda, 0\rangle$ \\
\hline $0111 \mapsto 01111111$ & $1000 \mapsto 10000000$ & $\langle 00,0\rangle \mapsto\langle 00,0\rangle\langle\lambda, 1\rangle$ \\
\hline
\end{tabular}

4.2 Case $w=10^{k-2} 1$

Theorem 4.2.1 Let $w=10^{k-2} 1, k \geq 3$ 周. Then $t_{w}=\prod_{i=0}^{\infty} w_{i}$ where

$$
\begin{aligned}
w_{0} & =0^{2^{k-1}+1} 10^{2^{k-1}} 1^{2} 0^{2^{k-1}-3} 1 ; \\
w_{1} & = \begin{cases}0^{6} 101^{2} 0^{2} 1^{2} 01 & \text { if } k=3 ; \\
0^{2^{k-1}+2} 1^{4} 0^{2^{k-1}-7} 10^{2^{k-1}} 1^{2} 0^{2^{k-1}-3} 1 & \text { if } k>3 ;\end{cases} \\
w_{i+1} & =0^{2^{k-1}-2} \mu\left(\left(0^{2^{k-1}-2}\right)^{-1} w_{i} 0^{2^{k-1}-2}\right)\left(0^{2^{k-1}-2}\right)^{-1} \quad \text { for } \quad i \geq 1 .
\end{aligned}
$$

For $i \geq 0$, the word $w_{i}$ is a Lyndon word and $w_{i}>w_{i+1}$.

Example 4.2.2 $w=1001 \quad(k=4)$

The case $k=2$ yields a different Lyndon factorization—see Section 4.3 . 


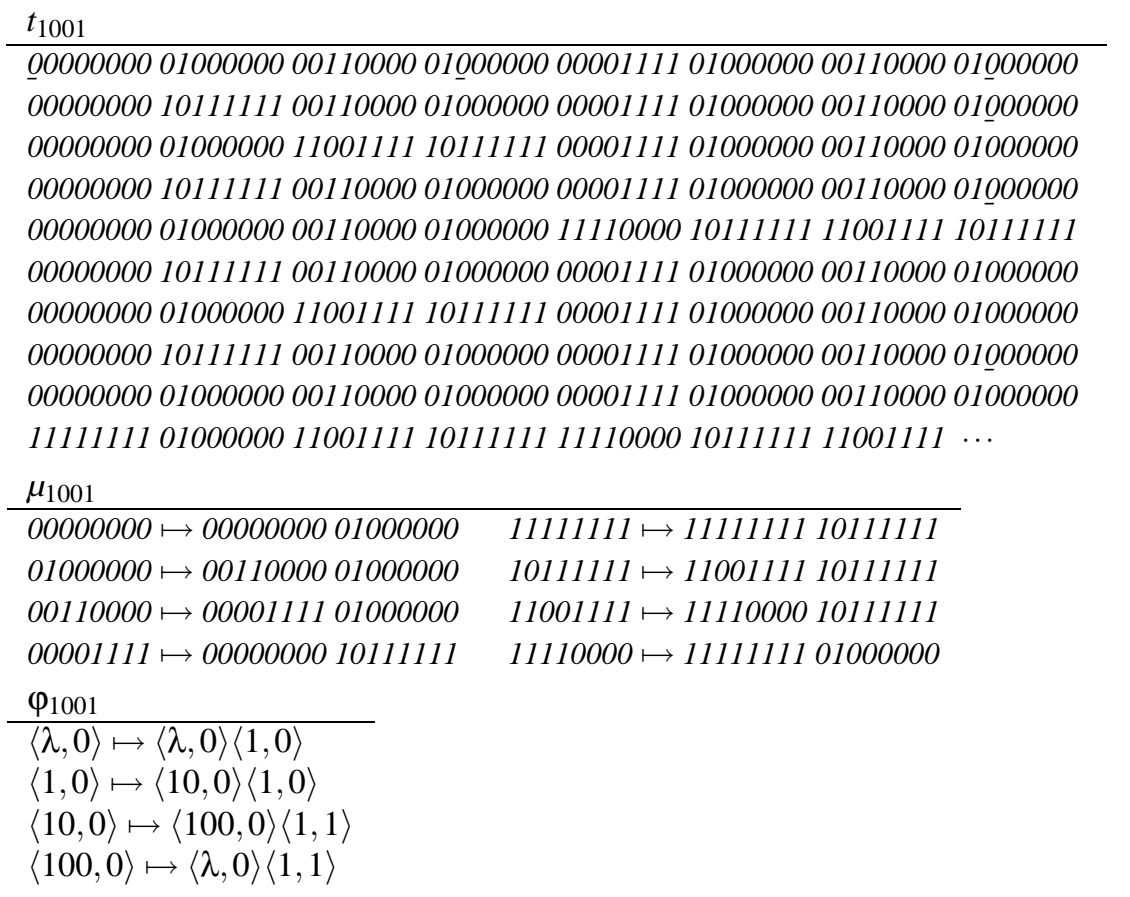

\subsection{Case $w=1^{k}$.}

Theorem 4.3.1 Let $w=1^{k}, k \geq 2$. Then $t_{w}=\prod_{i=0}^{\infty} w_{i}$ where $w_{0}=0^{2^{k}-1} 10^{2^{k-2}} 1$, $w_{1}=w_{0}^{-1} \mu^{2}\left(w_{0} 0\right) 0^{-1}$ and $w_{i+1}=0 \mu^{2}\left(0^{-1} w_{i} 0\right) 0^{-1}$ for $i \geq 1$.

For $i \geq 0$, the word $w_{i}$ is a Lyndon word and $w_{i}>w_{i+1}$.

Example 4.3.2 $w=11 \quad(k=2)$

$t_{11}$

o0010010000111010001001011100010 00010010000111011110110100011101 0001001000011101000100101110001011101101111000100001001011100010 0001001000011101000100101110001000010010000111011110110100011101 1110110111100010111011010001110100010010000111011110110100011101 0001001000011101000100101110001000010010000111011110110100011101 0001001000011101000100101110001011101101111000100001001011100010 1110110111100010111011010001110111101101111000100001001011100010 $000100100001110100010010111000101110110111100010000100101110001 \underline{0}$ 0001001000011101000100101110001000010010000111011110110100011101 $00010010000111010001001011100010111011011110001000010010 \ldots$

\begin{tabular}{ll}
$\mu_{11}$ & \\
\hline $00 \mapsto 0001$ & $11 \mapsto 1110$ \\
$01 \mapsto 0010$ & $10 \mapsto 1101$
\end{tabular}

$$
\begin{aligned}
& \varphi_{11} \\
& \hline\langle\lambda, 0\rangle \mapsto\langle\lambda, 0\rangle\langle 1,0\rangle \\
& \langle 1,0\rangle \mapsto\langle\lambda, 0\rangle\langle 1,1\rangle
\end{aligned}
$$




\subsection{Case $w=10^{k-1}$}

Theorem 4.4.1 Let $w=10^{k-1}, k \geq 2$. Denote

$$
\begin{aligned}
\rho & =\mu^{2 k-2}\left(1^{2^{k-1}}\right) \mu^{k-1}\left(0^{2^{k-1}}\right)\left(0^{2^{k-1}-1}\right)^{-1}, \\
j_{0} & =0, \\
j_{1} & =\left[10^{k-1} 10^{2 k-2}\right]_{2} \\
j_{2} & =\left[10^{k} 10^{2 k-2}\right]_{2},
\end{aligned}
$$

and, for $1 \leq r \leq k-1$, denote

$$
\begin{aligned}
& j_{2 r+1}=\left[10^{k-1} 10^{k-1} 1^{r} 0^{k-2} 1\right]_{2}, \\
& j_{2 r+2}=\left[10^{k} 10^{k-1} 1^{r} 0^{k-2} 1\right]_{2} .
\end{aligned}
$$

For $0 \leq i \leq 2 k-1$, let $w_{i}$ be the factor of $t_{w}$ of length $j_{i+1}-j_{i}$ at the position $j_{i}$ and, for $i \geq 2 k$, let $w_{i}=\rho^{-1} \mu\left(\rho w_{i-1} \rho^{-1}\right) \rho$.

Then $t_{w}=\prod_{i=0}^{\infty} w_{i}$ and, for $i \geq 0, w_{i}>w_{i+1}$, and $w_{i}$ is a Lyndon word.

Example 4.4.2 $w=100(k=3)$

$t_{100}$
0000100011001000111110001100100011110111110010001111100011001000
1111011100110111111110001100100011110111110010001111100011001000
1111011100110111000001110011011111110111110010001111100011001000
1111011100110111111110001100100011110111110010001111100011001000
1111011100110111000001110011011100001000001101110000011100110111
1111011100110111111110001100100011110111110010001111100011001000
1111011100110111000001110011011111110111110010001111100011001000
1111011100110111111110001100100011110111110010001111100011001000
1111011100110111000001110011011100001000001101110000011100110111
$00001000110010000000011100110111000010000011011100000111 \cdots$

\begin{tabular}{llll}
$\mu_{100}$ & & $\varphi_{100}$ \\
\hline $0000 \mapsto 00001000$ & $1111 \mapsto 11110111$ & & $\langle\lambda, 0\rangle \mapsto\langle\lambda, 0\rangle\langle 1,0\rangle$ \\
$1000 \mapsto 11001000$ & $0111 \mapsto 00110111$ & & $\langle 1,0\rangle \mapsto\langle 10,0\rangle\langle 1,0\rangle$ \\
$1100 \mapsto 11111000$ & $0011 \mapsto 00000111$ & & $\langle 10,0\rangle \mapsto\langle\lambda, 1\rangle\langle 1,0\rangle$
\end{tabular}

\section{Proofs of the factorization results.}

The present part contains proofs of the four theorems from Section 3. In each of the four cases, we start by observing some specific properties of $\varphi_{w}, \Psi_{w}, \mathfrak{A}_{w}$ and $\mu_{w}$ for the particular shape of $w$. The proof of each of the theorems consists of the following parts: 
Part A. We prove the consistency of the recurrent formula for factors $w_{i}$ and we show that the sequence $t_{w}$ can be written as a concatenation of the factors $w_{i}$.

Part B. We prove that $w_{0}, w_{1}, \ldots$ is a decreasing sequence.

Part $\boldsymbol{C}$. We show that each word $w_{i}$ is a Lyndon word-separately for the preamble and for the body of the factorization.

\subsection{Case $w=0^{k-1} 1$}

Let $w=0^{k-1} 1, k \geq 2$. In this case $s=0, \Psi=\psi \circ \varphi^{k-1}$ and each element of $\Sigma$ is of the form $\left\langle 0^{r}, m\right\rangle$ for some $r \in[k], m \in[2]$. The initial state of $\mathfrak{A}$ is $\left\langle 0^{k-1}, 0\right\rangle$.

We have a simple equivalent characterization of the order relation on $\Sigma$ for this case.

Property 5.1.1 Let $a, b \in \Sigma, a=\left\langle 0^{p}, m\right\rangle, b=\left\langle 0^{q}, n\right\rangle$. Then $a<b$ iff one of the following is true:

1. $m<n$ or

2. $m=n=0$ and $p<q$ or

3. $m=n=1$ and $p>q$.

Proof. If $0=m<n=1$ then $\psi\left(\delta^{*}\left(a, 0^{k-1}\right)\right)=0, \psi\left(\delta^{*}\left(b, 0^{k-1}\right)\right)=1$, and $a<b$. Now let $m=n$. For $r \in\{p, q\}, i \in\left[2^{k-1}\right]$ we have

$$
\Psi\left(\left\langle 0^{r}, m\right\rangle\right)(i)=\psi\left(\delta^{*}\left(\left\langle 0^{r}, m\right\rangle, i_{[2], k-1}\right)\right)= \begin{cases}m & \text { if } i=0 \text { or } i \geq 2^{r} \\ 1-m & \text { for } 1 \leq i<2^{r}\end{cases}
$$

Thus $\Psi\left(\left\langle 0^{r}, m\right\rangle\right)$ has the prefix $m(1-m)^{2^{r}-1}$. If $m=0$ then $a<b$ iff $p<q$, if $m=1$ then $a<b$ iff $p>q$.

The following complete description of the mapping $\varphi$ can be easily obtained using Remark 2.1.2.

Property 5.1.2 $\varphi\left(\left\langle 0^{r}, 0\right\rangle\right)=\left\langle 0^{r+1}, 0\right\rangle\langle\lambda, 0\rangle$ for $0 \leq r<k-1$, and $\varphi\left(\left\langle 0^{k-1}, 0\right\rangle\right)=\left\langle 0^{k-1}, 0\right\rangle\langle\lambda, 1\rangle$.

The first of the next two properties may be obtained, considering Remark 2.1.2, by observing the shapes of the words $i_{[2], k-1}, i \in\left[2^{k-1}\right]$. The second is a consequence of the trivial fact that the binary representation of a position number has suffix 0 iff the position number is even.

Property 5.1.3 Let $a \in \Sigma$. If $0^{2}$ is a prefix of $\Psi(a)$ then $a=\langle\lambda, 0\rangle$. If $0^{2^{k-1}-1}$ is a suffix of $\Psi(a)$ then $a=\langle\lambda, 0\rangle$ or $a=\left\langle 0^{k-1}, 1\right\rangle$.

Property 5.1.4 Let $x \in \Sigma^{*}$. Then $\langle\lambda, 0\rangle$ does not occur in any even position of $\varphi(x)$ and $\left\langle 0^{k-1}, 1\right\rangle$ does not occur in any odd position of $\varphi(x)$.

$\S$ The case $k=1$ corresponds to the sequence of Thue. Though the factorization in this case (see []]) has the same shape as for $k \geq 2$, the proof for $k \geq 2$ will use the fact that $w$ contains at least one symbol 0 . 


\section{Proof of Theorem 4.1 .1}

\section{Part $A$}

Lemma 5.1.5 $w_{0} w_{1}$ is a prefix of $t_{w}$.

Proof. Observing the shape of the words $r_{[2], k+2}$, for $0 \leq r \leq 2^{k+1}$, we find

$$
\psi\left(\delta^{*}\left(\left\langle 0^{k-1}, 0\right\rangle, r_{[2], k+2}\right)\right)=\left\{\begin{array}{ll}
0 & \text { if } r=0 \\
1 & \text { if } 0<r \leq 2^{k} \\
0 & \text { if } r=2^{k}+1 \\
1 & \text { if } 2^{k}+1<r \leq 2^{k+1}
\end{array} .\right.
$$

Therefore $t_{w}$ has the prefix $01^{2^{k}} 01^{2^{k}-1}$.

Lemma 5.1.6 For $i \geq 1,11$ is a suffix of $w_{i}$ and $1 w_{i} 1^{-1}=\Psi\left(\varphi^{i}(\langle\lambda, 1\rangle)\right.$.

Proof. The assertion is true for $i=1$ since $w_{1}=01^{2^{k}-1}$ and $1 w_{1} 1^{-1}$ is a factor of $t_{w}$ at position $2^{k}$, as implied by Lemma 5.1.5. Assume that the assertion is true for some $i \geq 1$. Then 1 is both a prefix and a suffix of $1 w_{i} 1^{-1}$. Theorem 2.3.2 implies that 1 is both a prefix and a suffix of $\mu\left(1 w_{i} 1^{-1}\right)$ since $w_{[2]}$ is neither a suffix of $0_{[2], k+i}$ nor of $\left(2^{k+i}-1\right)_{[2], k+i}$. Hence 11 is a suffix of $w_{i+1}=1^{-1} \mu\left(1 w_{i} 1^{-1}\right) 1$. Using Property 3.2.1 we obtain

$$
1 w_{i+1} 1^{-1}=11^{-1} \mu\left(1 w_{i} 1^{-1}\right) 11^{-1}=\mu\left(1 w_{i} 1^{-1}\right)=\mu\left(\Psi\left(\varphi^{i}(\langle\lambda, 1\rangle)\right)=\Psi\left(\varphi^{i+1}(\langle\lambda, 1\rangle) .\right.\right.
$$

Lemma 5.1.7 $t_{w}=\prod_{i=0}^{\infty} w_{i}$.

Proof. Since $t_{w}$ is a fixed point of $\mu$, the image in $\mu$ of the prefix $01^{2^{k}-1}$ of length $2^{k}$ of $t_{w}$ is the prefix of length $2^{k+1}$ of $t_{w}$. Then

$$
\begin{aligned}
\mu\left(\prod_{i=0}^{\infty} w_{i}\right) & =\mu\left(01^{2^{k}} \prod_{i=1}^{\infty} w_{i}\right) \\
& =\mu\left(01^{2^{k}-1} \prod_{i=1}^{\infty}\left(1 w_{i} 1^{-1}\right)\right) \\
& \left.=\mu\left(01^{2^{k}-1}\right) \prod_{i=1}^{\infty} \mu\left(1 w_{i} 1^{-1}\right)\right) \\
& \left.=\left(w_{0} w_{1} 1^{-1}\right) \prod_{i=1}^{\infty} \mu\left(1 w_{i} 1^{-1}\right)\right) \\
& =\left(w_{0} w_{1} 1^{-1}\right) \prod_{i=1}^{\infty}\left(1 w_{i+1} 1^{-1}\right) \\
& =\prod_{i=0}^{\infty} w_{i} .
\end{aligned}
$$

Both sequences $t_{w}$ and $\prod_{i=0}^{\infty} w_{i}$ are fixed points of $\mu$ starting by the same prefix of length $2^{k-1}$, therefore $t_{w}=\prod_{i=0}^{\infty} w_{i}$.

\section{Part $B$}

Lemma 5.1.8 $\left(w_{i}\right)_{i=0}^{\infty}$ is a decreasing sequence of words.

Proof. Clearly $w_{0}>w_{1}$. Property 5.1 .1 implies that $\langle\lambda, 1\rangle>\langle 0,1\rangle\langle\lambda, 1\rangle=\varphi(\langle\lambda, 1\rangle)$. Since $\varphi$ an $\Psi$ are strictly growing, we get, for $i \geq 1, \varphi^{i}(\langle\lambda, 1\rangle)>\varphi^{i+1}(\langle\lambda, 1\rangle)$, and, consequently,

$$
w_{i}=1^{-1} \Psi\left(\varphi^{i}(\langle\lambda, 1\rangle)\right) 1>1^{-1} \Psi\left(\varphi^{i+1}(\langle\lambda, 1\rangle)\right) 1=w_{i+1}
$$




\section{Part C}

We start by observing the fact that $w_{0}$ is strictly smaller than any of its non-empty proper suffixes.

Observation 5.1.9 The word $w_{0}$ is a Lyndon word.

Lemma 5.1.10 For $1 \leq i \leq k, w_{i}=0^{2^{i}-1} 1^{2^{k}-2^{i}} x_{i}$, where $x_{i}$ does not contain a factor $0^{2^{i}-1}$

Proof. For $0 \leq r \leq k-2, \varphi\left(\left\langle 0^{r}, 0\right\rangle\right)=\left\langle 0^{r+1}, 0\right\rangle\langle\lambda, 0\rangle$ and (for $\left.r=k-1\right) \varphi\left(\left\langle 0^{k-1}, 0\right\rangle\right)=\left\langle 0^{k-1}, 0\right\rangle\langle\lambda, 1\rangle$. An easy induction yields, for $0 \leq i \leq k-1$,

$$
\psi\left(\varphi^{i}(\langle\lambda, 0\rangle)\right)=\psi\left(\left\langle 0^{i}, 0\right\rangle\langle\lambda, 0\rangle \varphi(\langle\lambda, 0\rangle) \varphi^{2}(\langle\lambda, 0\rangle) \cdots \varphi^{i-1}(\langle\lambda, 0\rangle)\right)=0^{2^{i}} .
$$

For $1 \leq i \leq k$,

$$
\psi\left(\varphi^{k-1+i}(\langle\lambda, 1\rangle)\right)=x y
$$

where

$$
\begin{aligned}
x & =\psi\left(\left\langle 0^{k-1}, 1\right\rangle\langle\lambda, 0\rangle \varphi(\langle\lambda, 0\rangle) \cdots \varphi^{i-1}(\langle\lambda, 0\rangle) \varphi^{i}(\langle\lambda, 1\rangle) \cdots \varphi^{k-1}(\langle\lambda, 1\rangle)\right) \\
& =10^{2^{0}+2^{1}+\cdots 2^{(i-1)}} 1^{2^{i}+2^{i+1}+\cdots 2^{k-1}}
\end{aligned}
$$

and

$$
\begin{aligned}
y & =\Psi\left(\varphi^{k-1+1}(\langle\lambda, 1\rangle) \cdots \varphi^{k-1+i-1}(\langle\lambda, 1\rangle)\right) \\
& =\Psi(\varphi(\langle\lambda, 1\rangle)) \cdots \Psi\left(\varphi^{i-1}(\langle\lambda, 1\rangle)\right) .
\end{aligned}
$$

Hence, following Lemma 5.1.6,

$$
\begin{aligned}
w_{i} & =1^{-1} \psi\left(\varphi^{k-1+i}(\langle\lambda, 1\rangle)\right) 1 \\
& =0^{2^{i}-1} 1^{2^{k}-2^{i}}\left(1 w_{1} 1^{-1}\right) \cdots\left(1 w_{i-1} 1^{-1}\right) 1 \\
& =0^{2^{i}-1} 1^{2^{k}-2^{i}} w_{1} \cdots w_{i-1},
\end{aligned}
$$

where $w_{1} \cdots w_{i-1}$ does not contain a factor $0^{2^{i}-1}$, the latter fact follows by induction.

Corollary 5.1.11 $w_{1}, \ldots, w_{k}$ are Lyndon words

Lemma 5.1.12 For $i \geq k+1,0^{2^{k}}$ is a prefix of $w_{i}$.

Proof. It is enough to prove the assertion for $w_{k+1}$, since, by Lemma 5.1.8, for $i>k+1, w_{k+1}>w_{i}$. From Lemma 5.1.6 we obtain

$$
\begin{aligned}
w_{k+1} & =1^{-1} \Psi\left(\varphi^{k+1}(\langle\lambda, 1\rangle)\right) 1 \\
& =1^{-1} \Psi\left(\left\langle 0^{k-1}, 1\right\rangle\langle\lambda, 0\rangle\langle 0,0\rangle x\right) 1 \\
& =1^{-1} 10^{2^{k-1}-1} 0^{2^{k-1}} 010^{2^{k-1}-2} \Psi(x)=0^{2^{k}} y
\end{aligned}
$$

where $x \in \Sigma^{*}, y \in[2]^{*}$ are suitable words.

Lemma 5.1.13 For $i \geq 1, w_{1}$ is a primitive word. 
Proof. Denote $\omega=\Psi\left(\varphi^{i}(\langle\lambda, 1\rangle)\right)$. By Lemma 5.1.6, $w_{i}=1^{-1} \omega 1$. Assume that $w_{i}$ is not primitive. Then neither is $\omega$. Since the length of $\omega$ is a positive power of $2, \omega$ is a concatenation of two equal factors, implying $\varphi\left(b_{1}\right)=a a$ for some symbol $a \in \Sigma$ - a contradiction to Proposition 2.3.5.

Lemma 5.1.14 For $i \geq k, w_{i}$ is a Lyndon word.

Proof. By induction. For $i=k$ the assertion follows from Corollary 5.1.11. Let $i>k$. Assume that $w_{i-1}$ is a Lyndon word. By Lemma 5.1.13, $w_{i}$ is not primitive. Assume that $w_{i}$ is not minimal in its conjugacy class. Let $w_{i}=u v$ where $v u<w_{i}$ is the smallest conjugate of $w_{i}$. The minimality of $v u$ implies that the last symbol of $u$ is 1 . The word $v u$, as a conjugate of $\omega$, is of the form $\xi_{0} x_{1} x_{2} y \xi_{0}^{\prime}, \xi_{0} \neq \lambda$, where $\xi_{0}^{\prime} \xi_{0}=\Psi\left(c_{0}\right)$, $x_{1}=\Psi\left(c_{1}\right), x_{2}=\Psi\left(c_{2}\right)$ for some $c_{0}, c_{1}, c_{2} \in \Sigma$ and $\xi_{0}^{\prime} \xi_{0} x_{1} x_{2} y=\Psi\left(c_{0} c_{1} c_{2} z\right)$ for some conjugate $c_{0} c_{1} c_{2} z$ of $\varphi^{i}(\langle\lambda, 1\rangle)$. Since $0^{2^{k}}$ is a prefix of $w_{i}, 0^{2^{k}}$ must be a prefix of $v u$. The last symbol of $v$ is 1 (the last symbol of $w_{i}$ ) therefore $0^{2^{k}}$ is a prefix of $v$. Hence $\xi_{0} x_{1} \in 0^{*}$, and, consequently, $x_{1}=0^{2^{k-1}}$ and $c_{1}=\langle\lambda, 0\rangle$.

Property 5.1 .4 implies that $c_{0} \neq\langle\lambda, 0\rangle, c_{2} \neq\langle\lambda, 0\rangle$, otherwise $\langle\lambda, 1\rangle$ occurs in two neighbor positions. As a consequence of Property 5.1.3, $x_{1} x_{2}$ has the prefix $0^{2^{k-1}+1} 1$ or $0^{2^{k-1}} 1$. Therefore $\xi_{0}$ has the suffix $0^{2^{k-1}-1}$ and, again by Property 5.1.3, $c_{0}=\left\langle 0^{k-1}, 1\right\rangle$ and $\xi_{0}^{\prime}=1$. We have

$$
\Psi\left(c_{0} c_{1} c_{2} z\right)=\xi_{0}^{\prime} \xi_{0} x_{1} x_{2} y=1 v u 1^{-1}<1 w_{i} 1^{-1}=\Psi\left(\varphi^{i}(\langle\lambda, 1\rangle)\right)
$$

yielding $c_{0} c_{1} c_{2} z<\varphi^{i}(\langle\lambda, 1\rangle)$.

The word $c_{0} c_{1} c_{2} z=\left\langle 0^{k-1}, 1\right\rangle\langle\lambda, 0\rangle c_{2} z$ is a conjugate of $\varphi^{i}(\langle\lambda, 1\rangle)$, hence either $\left\langle 0^{k-1}, 1\right\rangle\langle\lambda, 0\rangle c_{2} z$ or $\langle\lambda, 0\rangle c_{2} z\left\langle 0^{k-1}, 1\right\rangle$ is an image in $\varphi$ of some word from $\Sigma^{*}$, the latter case contradicting Property 5.1.4.

Thus $\left\langle 0^{k-1}, 1\right\rangle\langle\lambda, 0\rangle c_{2} z=\varphi\left(z^{\prime}\right)$ for some conjugate $z^{\prime}$ of $\varphi^{i-1}(\langle\lambda, 1\rangle)$ where the initial symbol of $z^{\prime}$ is either $\left\langle 0^{k-1}, 1\right\rangle$ or $\left\langle 0^{k-2}, 1\right\rangle$, in both cases the first symbol of $\Psi\left(z^{\prime}\right)$ is 1 . Since $\varphi\left(z^{\prime}\right)<\varphi^{i}(\langle\lambda, 1\rangle)$, we have $z^{\prime}<\varphi^{i-1}(\langle\lambda, 1\rangle)$ and $\Psi\left(z^{\prime}\right)<\Psi\left(\varphi^{i-1}(\langle\lambda, 1\rangle)\right)$ implying

$$
1^{-1} \Psi\left(z^{\prime}\right) 1<1^{-1} \Psi\left(\varphi^{i-1}(\langle\lambda, 1\rangle)\right) 1=w_{i-1}
$$

where the left-hand side is a conjugate of $w_{i-1}$ - a contradiction to the inductive hypothesis that $w_{i-1}$ is a Lyndon word.

Theorem 4.1.1] now follows from Lemma 5.1.7, Lemma 5.1.8, Observation 5.1.9, Corollary 5.1.11] and Lemma 5.1.14.

\subsection{Case $w=10^{k-2} 1$}

Let $w=10^{k-2} 1, k \geq 3$. In this case $s=0, \Psi=\psi \circ \varphi^{k-1}$ and each element of $\Sigma$ is of the form $\left\langle 10^{r}, m\right\rangle$ for some $r \in[k-1], m \in[2]$. The initial state of $\mathfrak{A}$ is $\langle\lambda, 0\rangle$.

The first of the following two properties may be observed applying Remark 2.1.2. The second one follows from the fact that the only proper prefix of $w$ finished by 1 is 1 .

Property 5.2.1 for $r \in[k-1]$,

$$
\psi\left(\varphi^{r}(\langle\lambda, 0\rangle)\right)=\psi\left(\varphi^{r}(\langle 1,0\rangle)\right)=0^{2^{r}}
$$

and

$$
\begin{aligned}
\Psi(\langle\lambda, 0\rangle) & =0^{2^{k-1}}, \\
\Psi\left(\left\langle 10^{r}, 0\right\rangle\right) & =0^{2^{r}} 1^{2^{r}} 0^{2^{k-1}-2^{r+1}} .
\end{aligned}
$$


Property 5.2.2 If a symbol $a \in \Sigma$ occurs in an odd position of a word of the form $\varphi(x), x \in \Sigma^{*}$, then $a=\langle 1,0\rangle$ or $a=\langle 1,1\rangle$.

\section{Proof of Theorem 4.2 .1}

\section{Part $A$}

Lemma 5.2.3 $w_{0} w_{1}$ is a prefix of $t_{w}$ and $w_{1}=0^{2^{k-1}-2} \Psi\left(\varphi^{2}(\langle 1,0\rangle)\right)\left(0^{2^{k-1}-2}\right)^{-1}$.

Proof. The sequence $t_{w}$ has the prefix

$$
\Psi\left(\varphi^{3}(\langle\lambda, 0\rangle)\right)= \begin{cases}\Psi(\langle\lambda, 0\rangle\langle 1,0\rangle\langle 10,0\rangle\langle 1,0\rangle\langle\lambda, 0\rangle\langle 1,1\rangle\langle 10,0\rangle\langle 1,0\rangle) & \text { if } k=3 \\ \Psi(\langle\lambda, 0\rangle\langle 1,0\rangle\langle 10,0\rangle\langle 1,0\rangle\langle 100,0\rangle\langle 1,0\rangle\langle 10,0\rangle\langle 1,0\rangle) & \text { if } k>3\end{cases}
$$

In both cases Property 5.2.1 implies

$$
\begin{aligned}
\Psi\left(\varphi^{2}(\langle\lambda, 0\rangle\langle 1,0\rangle)\right) & =w_{0} w_{1} 0^{2^{k-1}-2} \quad \text { and } \\
\left(0^{2^{k-1}-2}\right)^{-1} w_{1} 0^{2^{k-1}-2} & =\Psi\left(\varphi^{2}(\langle 1,0\rangle)\right)
\end{aligned}
$$

since the latter word is a factor of $t_{w}$ of the length $2^{k+1}$ starting at position $2^{k+1}$.

Lemma 5.2.4 For $i \geq 1,0^{2^{k-1}-2}$ is a suffix of $\mu\left(\left(0^{2^{k-1}-2}\right)^{-1} w_{i} 0^{2^{k-1}-2}\right)$ and

$$
w_{i}=0^{2^{k-1}-2} \Psi\left(\varphi^{i+1}(\langle 1,0\rangle)\right)\left(0^{2^{k-1}-2}\right)^{-1} .
$$

Proof. Theorem 2.3.2 implies that the string $\mu\left(x 0^{2^{k-1}-2}\right)$, where $x 0^{2^{k-1}-2}$ is a string of a length, which is a multiple of $2^{k-1}$, ends in a string of length $2^{k}-4$ containing 0 in all positions $i$ except those where $i_{[2],[\log i]}$ ends in $w$. This is not true for the last $2^{k-1}-2$ position numbers, therefore 0 occurs in these positions. The proof of the latter part of the assertion of the lemma is a simple induction where Lemma 5.2 .3 provides the basic step and Property 3.2.1 is applied in the inductive step.

Lemma 5.2.5 $t_{w}=\prod_{i=0}^{\infty} w_{i}$.

Proof. Since $t_{w}$ is a fixed point of $\mu$, the image in $\mu$ of the prefix $w_{0} 0^{2^{k-1}-2}$ of length $2^{k+1}$ of $t_{w}$ is the prefix $w_{0} w_{1} 0^{2^{k-1}-2}$ of length $2^{k+2}$ of $t_{w}$. Applying Lemma 5.2.4 and Property 3.2.1 we obtain

$$
\begin{aligned}
\mu\left(\prod_{i=0}^{\infty} w_{i}\right) & =\mu\left(w_{0} 0^{2^{k-1}-2} \prod_{i=1}^{\infty}\left(0^{2^{k-1}-2}\right)^{-1} w_{i} 0^{2^{k-1}-2}\right) \\
& =\mu\left(w_{0} 0^{2^{k-1}-2} \prod_{i=1}^{\infty} \Psi\left(\varphi^{i+1}(\langle 1,0\rangle)\right)\right) \\
& =\mu\left(w_{0} 0^{2^{k-1}-2}\right) \prod_{i=1}^{\infty} \mu\left(\Psi\left(\varphi^{i+1}(\langle 1,0\rangle)\right)\right) \\
& =w_{0} w_{1} 0^{2^{k-1}-2} \prod_{i=1}^{\infty} \Psi\left(\varphi^{i+2}(\langle 1,0\rangle)\right) \\
& \left.=\left(w_{0} w_{1} 0^{2^{k-1}-2}\right) \prod_{i=1}^{\infty}\left(0^{2^{k-1}-2}\right)^{-1} w_{i+1} 0^{2^{k-1}-2}\right) \\
& =\prod_{i=0}^{\infty} w_{i}
\end{aligned}
$$

Both sequences $t_{w}$ and $\prod_{i=0}^{\infty} w_{i}$ are fixed points of $\mu$ starting by the same prefix of length $2^{k-1}$, therefore $t_{w}=\prod_{i=0}^{\infty} w_{i}$. 


\section{Part $B$}

Lemma 5.2.6 For $i \geq 0, w_{i}>w_{i+1}$.

Proof. Clearly, $w_{0}>w_{1}$. Let $i \geq 1$. Property 5.2.1 implies that

$$
\Psi(\langle 1,0\rangle)>\Psi(\langle 10,0\rangle),
$$

therefore

$$
\langle 1,0\rangle>\langle 10,0\rangle\langle 1,0\rangle=\varphi(\langle 1,0\rangle)
$$

and Lemma 5.2 .4 implies

$$
w_{i}=0^{2^{k-1}-2} \Psi\left(\varphi^{i+1}(\langle 1,0\rangle)\right)\left(0^{2^{k-1}-2}\right)^{-1}>0^{2^{k-1}-2} \Psi\left(\varphi^{i+2}(\langle 1,0\rangle)\right)\left(0^{2^{k-1}-2}\right)^{-1}=w_{i+1} .
$$

\section{Part C}

Lemma 5.2.7 For $i \in[k-1]$, $w_{i}$ is a Lyndon word.

Proof. Applying Property 5.2.1, for $2 \leq i \leq k-2$, we obtain

$$
\begin{aligned}
\left(0^{2^{k-1}-2}\right)^{-1} w_{i} 0^{2^{k-1}-2} & =\Psi\left(\varphi^{i+1}(\langle 1,0\rangle)\right) \\
& =\psi\left(\varphi^{k+i}(\langle 1,0\rangle)\right) \\
& =\Psi\left(\varphi^{i+2}\left(\left\langle 10^{k-2}, 0\right\rangle\langle 1,0\rangle \varphi(\langle 1,0\rangle) \cdots \varphi^{k-3}(\langle 1,0\rangle)\right)\right) \\
& =\psi\left(\varphi^{i+1}\left(\langle\lambda, 0\rangle\langle 1,1\rangle \varphi(\langle 1,0\rangle) \cdots \varphi^{k-2}(\langle 1,0\rangle)\right)\right) .
\end{aligned}
$$

If $i<k-2$ then

$$
\begin{aligned}
\left(0^{2^{k-1}-2}\right)^{-1} w_{i} 0^{2^{k-1}-2} & =0^{2^{i+1}} 1^{2^{i+1}} \psi\left(\varphi^{i+2}(\langle 1,0\rangle) \cdots \varphi^{k+i-1}(\langle 1,0\rangle)\right) \\
& =0^{2^{i+1}} 1^{2^{i+1}} x y z
\end{aligned}
$$

where

$$
\begin{aligned}
x & =\psi\left(\varphi^{i+2}(\langle 1,0\rangle) \cdots \varphi^{k-2}(\langle 1,0\rangle)\right) \\
& =0^{2^{i+2}+\cdots+2^{k-2}} \\
& =0^{2^{k-1}-2^{i+2}} \\
y & =\Psi\left(\varphi^{k-1}(\langle 1,0\rangle) \varphi^{k}(\langle 1,0\rangle)\right) \\
& =\Psi(\langle 1,0\rangle\langle 10,0\rangle\langle 1,0\rangle) \\
& =\left(010^{2^{k-1}-2}\right)\left(0^{2} 1^{2} 0^{2^{k-1}}\right)\left(010^{2^{k-1}-2}\right) \\
z & =\psi\left(\varphi^{k+1}(\langle 1,0\rangle) \cdots \varphi^{k+i-1}(\langle 1,0\rangle)\right) \\
& =\left(0^{2^{k-1}-2}\right)^{-1} w_{1} \cdots w_{i-1} 0^{2^{k-1}-2}
\end{aligned}
$$

and, consequently, $w_{i}=0^{2^{k-1}+2^{i+1}-2} 1^{2^{i+1}} 0^{2^{k-1}} 10^{2^{k-1}} 1^{2} 0^{2^{k-1}+1} 1 w_{1} \cdots w_{i-1}$. If $i=k-2$ then

$$
\begin{aligned}
\left(0^{2^{k-1}-2}\right)^{-1} w_{i} 0^{2^{k-1}-2} & =\Psi\left(\langle\lambda, 0\rangle\langle 1,1\rangle \varphi(\langle 1,0\rangle) \varphi^{2}(\langle 1,0\rangle) \cdots \varphi^{k-2}(\langle 1,0\rangle)\right) \\
& =x y
\end{aligned}
$$


where

$$
\begin{aligned}
x & =\Psi(\langle\lambda, 0\rangle\langle 1,1\rangle \Psi(\varphi(\langle 1,0\rangle)) \\
& =\left(0^{2^{k-1}}\right)\left(101^{2^{k-1}-2}\right)\left(0^{2} 1^{2} 0^{2^{k-1}-4} 010^{2^{k-1}-2}\right) \\
y & =\Psi\left(\varphi^{2}(\langle 1,0\rangle)\right) \cdots \Psi\left(\varphi^{k-2}(\langle 1,0\rangle)\right) \\
& =\left(0^{2^{k-1}-2}\right)^{-1} w_{1} \cdots w_{i-1} 0^{2^{k-1}-2}
\end{aligned}
$$

and, consequently, $w_{i}=0^{2^{k}-2} 101^{2^{k-1}-2} 0^{2} 1^{2} 0^{2^{k-1}-3} 1 w_{1} \cdots w_{i-1}$.

In both cases, denote as $\alpha$ the prefix of $w_{i}$ satisfying $w_{i}=\alpha w_{1} \cdots w_{i-1}$. Let us now prove the assertion of the lemma by induction. Each of the words $w_{0}, w_{1}$ is smaller than any of its non-empty proper suffixes, therefore they are Lyndon words. Let us now assume that $w_{0}, \ldots, w_{i-1}, 2 \leq i \leq k-2$, are Lyndon words. If $\beta \neq \lambda$ is a proper suffix of $\alpha$ then $0^{2^{k}+2^{i+1}-2}$ is not a factor of $\beta$, hence $w_{i}<\beta<\beta w_{1} \cdots w_{i-1}$. If $\beta \neq \lambda$ is a proper suffix of some $w_{j}, j \in[i]$, then Lemma 5.4 .7 implies $w_{i}<w_{j}<\beta<\beta w_{1} \cdots w_{i-1}$ since $w_{j}$ is a Lyndon word. Moreover, $w_{i}<w_{j}<w_{j} \cdots w_{i-1}$ for $j \in[i]$. Therefore $w_{i}$ is a Lyndon word, since it is smaller than any of its non-empty proper suffixes.

Lemma 5.2.8 If $\langle\lambda, 0\rangle x \in \Sigma^{*}$ is a conjugate of a word $\varphi^{2}(y)$ for some $y \in \Sigma^{*}$ then $\langle\lambda, 0\rangle x=\varphi^{2}\left(y^{\prime}\right)$ for some conjugate $y^{\prime}$ of $y$ and the last symbol of $\varphi\left(y^{\prime}\right)$ is $\langle 1,0\rangle$.

Proof. Assume the smallest number $r, 0 \leq r \leq 2$, such that $\langle\lambda, 0\rangle x=\varphi^{2-r}(z)$ for some conjugate $z$ of $\varphi^{r}(y)$. If $r>0$ then, as implied by Property 5.2.2, $z$ starts either by $\langle 1,0\rangle$ or by $\langle 1,1\rangle$. Consequently, $\langle\lambda, 0\rangle x=\varphi^{2-r}(z)$ starts by $\left\langle 10^{2-r}, 0\right\rangle$ or by $\left\langle 10^{2-r}, 1\right\rangle$ - a contradiction. The second assertion of the lemma follows from the observation, that if, for some $a \in \Sigma, \varphi(a)$ ends in $\langle 1,0\rangle$ then either $a=\left\langle 10^{k-2}, 1\right\rangle$ or $a=\langle 1,0\rangle$. As follows from Property 5.2.2, the former case is not possible if $a$ is the last symbol of $\varphi\left(y^{\prime}\right)$.

Lemma 5.2.9 For $i \geq 1, w_{1}$ is a primitive word.

Proof. Analogous to the proof of Lemma 5.1.13.

Lemma 5.2.10 For $i \geq k-2, w_{i}$ is a Lyndon word.

Proof. We proceed by induction. For $i=k-2$ the assertion follows from Lemma 5.2.7.

Let $i \geq k-1$ and let $w_{i-1}$ be a Lyndon word. Lemma 5.2.9 states that $w_{i}$ is not primitive. Assume that $w_{i}$ is not minimal in its conjugacy class. Let $w_{i}=u v$ where $v u<w_{i}$ is the smallest conjugate of $w_{i}$ (and of $\omega$ ). The minimality of $v u$ implies that the last symbol of $u$ is 1 . The word $v u$, as a conjugate of $w_{i}$, is of the form $\xi_{0} x_{1} x_{2} y \xi_{0}^{\prime}, \xi_{0} \neq \lambda$, where $\xi_{0}^{\prime} \xi_{0}=\Psi\left(c_{0}\right), x_{1}=\Psi\left(c_{1}\right), x_{2}=\Psi\left(c_{2}\right)$ for some $c_{0}, c_{1}, c_{2} \in \Sigma$ and $\xi_{0}^{\prime} \xi_{0} x_{1} x_{2} y=\Psi\left(c_{0} c_{1} c_{2} z\right)$ for some conjugate $c_{0} c_{1} c_{2} z$ of $\varphi^{i+1}(\langle 1,0\rangle)$. The prefix of $\left(0^{2^{k-1}-2}\right)^{-1} w_{i}$ is $\Psi\left(\varphi^{i+1-(k-1)}(\langle\lambda, 0\rangle)\right)$ being a prefix of length $2^{i+1} \geq 2^{k}$ of $t_{w}$. Therefore $w_{i}$ has the prefix $0^{2^{k-1}-2} 0^{2^{k-1}+1} 1=0^{2^{k}-1} 1$. This must be a prefix of $v u$, as well.

Either $\xi_{0} x_{1} \in 0^{*}$ or $x_{1}=0^{2^{k-1}-1} 1$, the latter case is not possible due to Property 5.2.1, hence $x_{1}=0^{2^{k-1}}$ and $c_{1}=\langle\lambda, 0\rangle$. Property 5.2.2 implies that $c_{0} \neq\langle\lambda, 0\rangle$, and, again using Property 5.2.1, $c_{0}=\langle 1,0\rangle$, $\xi_{0}=0^{2^{k-1}-2}, \xi_{0}^{\prime}=01$, and $c_{2}=\langle 1,0\rangle$.

Following Lemma 5.2.8, $c_{1} c_{2} z c_{0}=\varphi^{2}\left(y^{\prime}\right)$ for some conjugate $y^{\prime}$ of $\varphi^{i-1}(\langle 1,0\rangle)$ where the last symbol of $\varphi^{i}\left(y^{\prime}\right)$ is $\langle 1,0\rangle$. We have

$$
\Psi\left(c_{1} c_{2} z c_{0}\right)=x_{1} x_{2} y \xi_{0}^{\prime} \xi_{0}=\xi_{0}^{-1} v u \xi_{0}<\xi_{0}^{-1} w_{i} \xi_{0}=\Psi\left(\varphi^{i+1}(\langle 1,0\rangle)\right)
$$


yielding $\varphi^{2}\left(y^{\prime}\right)=c_{1} c_{2} z c_{0}<\varphi^{i+1}(\langle 1,0\rangle)$ and $\varphi\left(y^{\prime}\right)<\varphi^{i}(\langle 1,0\rangle)$. Since the suffix $\langle 1,0\rangle$ is preserved by $\varphi$, the strings on both sides of the last inequality are finished by $\langle 1,0\rangle$, therefore $\Psi\left(\varphi\left(y^{\prime}\right)\right)<\Psi\left(\varphi^{i}(\langle 1,0\rangle)\right)$ and the strings on both sides of the last inequality are finished by $\Psi(\langle 1,0\rangle)=010^{2^{k-1}}-2$.

Hence

$$
0^{2^{k-1}-2} \Psi\left(\varphi\left(y^{\prime}\right)\right)\left(0^{2^{k-1}-2}\right)^{-1}<0^{2^{k-1}-2} \Psi\left(\varphi^{i}(\langle 1,0\rangle)\right)\left(0^{2^{k-1}-2}\right)^{-1}=w_{i-1}
$$

- a contradiction, since the left-hand side is a conjugate of $w_{i-1}$ and $w_{i-1}$ is a Lyndon word.

Theorem 4.1.1 now follows from Lemma 5.2.5, Lemma 5.2.6, Lemma 5.2.7 and Lemma 5.2.10.

\subsection{Case $w=1^{k}$.}

Let $w=1^{k}, k \geq 2$. In this case $s=0, \Psi=\psi \circ \varphi^{k-1}$ and each element of $\Sigma$ is of the form $\left\langle 1^{r}, m\right\rangle$ for some $r \in[k], m \in[2]$. The initial state of $\mathfrak{A}$ is $a_{0}=\langle\lambda, 0\rangle$.

The first of the following two properties follows from Remark 2.1.2. The second is based on the fact that the last symbol of each non-empty prefix of $w$ is 1 .

Property 5.3.1 for $r \in[k-1]$,

$$
\psi\left(\varphi^{r}(\langle\lambda, 0\rangle)\right)=\psi\left(\varphi^{r}(\langle 1,0\rangle)\right)=0^{2^{r}}
$$

and

$$
\begin{aligned}
& \Psi(\langle\lambda, 0\rangle)=0^{2^{k-1}}, \\
& \Psi(\langle 1,0\rangle)=0^{2^{k-1}-1} 1,
\end{aligned}
$$

and, for $k \geq 3, r \geq 2$,

$$
\Psi\left(\left\langle 1^{r}, 0\right\rangle\right)=0^{2^{k-1}-2^{r-1}} 1^{2^{r-2}} 0^{2^{r-3}} 1^{2^{r-4}} 0^{2^{r-5}} \cdots d^{2^{0}}(1-d)
$$

where $d=0$ if $r$ is odd and $d=1$ if $r$ is even.

Property 5.3.2 If a symbol $a \in \Sigma$ occurs in an even position of a word of the form $\varphi(x), x \in \Sigma^{*}$, then $a=\langle\lambda, 0\rangle$ or $a=\langle\lambda, 1\rangle$. No symbol from $\Sigma$ can occur both in an even and in an odd position of $\varphi(x)$.

\section{Proof of Theorem 4.3 .1}

\section{Part $A$}

Lemma 5.3.3 $w_{0} w_{1}$ is a prefix of $t_{w}, w_{1}=0 \Psi\left(\varphi^{2}(\langle 1,0\rangle) \varphi^{3}(\langle 1,0\rangle)\right) 0^{-1}$ and $0^{2^{k}} 1$ is a prefix of $w_{1}$.

Proof. Property 5.3.1 implies that

$$
w_{0}=\Psi(\langle\lambda, 0\rangle\langle 1,0\rangle\langle\lambda, 0\rangle\langle 1,1\rangle) 0^{-1} i \quad \text { if } k=2
$$

and

$$
w_{0}=\Psi(\langle\lambda, 0\rangle\langle 1,0\rangle\langle\lambda, 0\rangle\langle 11,0\rangle) 0^{-1} \quad \text { if } k \geq 3 .
$$


In both cases, $w_{0}=. \Psi\left(\varphi^{2}(\langle\lambda, 0\rangle)\right) 0^{-1}$. The sequence $t_{w}$ has the prefix $\Psi\left(\varphi^{2}(\langle\lambda, 0\rangle)\right)=w_{0} 0$. Using Property 3.2 .1 we obtain

$$
\begin{aligned}
w_{0} w_{1} 0 & =w_{0}\left[\left(w_{0}\right)_{0}^{-1} \mu^{2}\left(w_{0} 0\right)\right] \\
& =\mu^{2}\left(w_{0} 0\right) \\
& \left.=\Psi\left(\varphi^{4}(\langle\lambda, 0\rangle)\right)\right) \\
& =\Psi\left(\varphi^{2}(\langle\lambda, 0\rangle\langle 1,0\rangle)\right) \Psi\left(\varphi^{3}(\langle 1,0\rangle)\right) \\
& \left.=w_{0} 0 \Psi\left(\varphi^{2}\langle 1,0\rangle\right)\right) \Psi\left(\varphi^{3}(\langle 1,0\rangle)\right) .
\end{aligned}
$$

Thus $w_{0} w_{1}$ is a prefix of $t_{w}$,

$$
w_{1}=0 \Psi\left(\varphi ^ { 2 } \left(\langle 1,0\rangle \varphi^{3}(\langle 1,0\rangle) 0^{-1}\right.\right.
$$

and $w_{1}$ has the prefix

$$
0 \Psi(\langle\lambda, 0\rangle\langle 1,0\rangle)=00^{2^{k-1}} 0^{2^{k-1}-1} 1=0^{2^{k}} 1,
$$

as implied by Property 5.3.1.

Lemma 5.3.4 For $i \geq 1$,

$$
w_{i}=0 \Psi\left(\varphi^{2 i}(\langle 1,0\rangle) \varphi^{2 i+1}(\langle 1,0\rangle)\right) 0^{-1}
$$

and the last symbol of the word $\mu^{2}\left(0^{-1} w_{i-1} 0\right)$ is 0 .

Proof. Theorem 2.3.2 implies, that the last symbol of $\mu(x)$ is complementary to the last symbol of $x$. Therefore $\mu^{2}$ preserves the last symbol of its argument.

The assertion of the lemma is true for $i=1$ as follows from Lemma 5.3.3.

Now let the assertion be true for some $i-1 \geq 1$. Then

$$
\begin{aligned}
w_{i} & =0 \mu^{2}\left(0^{-1} w_{i-1} 0\right) 0^{-1} \\
& =0 \mu^{2}\left(0^{-1}\left(0 \Psi\left(\varphi^{2 i-2}(\langle 1,0\rangle) \varphi^{2 i-1}(\langle 1,0\rangle)\right) 0^{-1}\right) 0\right) 0^{-1} \\
& =0 \Psi\left(\varphi^{2}\left(\varphi^{2 i-2}(\langle 1,0\rangle) \varphi^{2 i-1}(\langle 1,0\rangle)\right)\right) 0^{-1} \\
& =0\left(\Psi\left(\varphi^{2 i}(\langle 1,0\rangle) \varphi^{2 i+1}(\langle 1,0\rangle)\right)\right) 0^{-1}
\end{aligned}
$$

Lemma 5.3.5 $t_{w}=\prod_{i=0}^{\infty} w_{i}$

Proof. It is enough to prove that the sequence $\prod_{i=0}^{\infty} w_{i}$, having the same prefix $w_{0} 0$ of length $2^{k+1}$ as $t_{w}$, is a fixed point of $\mu^{2}$. Applying Lemma 5.3.3 we obtain

$$
\begin{aligned}
\mu^{2}\left(\prod_{i=0}^{\infty} w_{i}\right) & =\mu^{2}\left(w_{0} 0 \prod_{i=1}^{\infty}\left(0^{-1} w_{i} 0\right)\right) \\
& =w_{0}\left(w_{0}^{-1} \mu^{2}\left(w_{0} 0\right) \prod_{i=1}^{\infty}\left(0^{-1} w_{i+1} 0\right)=\prod_{i=0}^{\infty} w_{i} .\right.
\end{aligned}
$$




\section{Part $B$}

Lemma 5.3.6 For $i \geq 0, w_{i}>w_{i+1}$.

Proof. By Lemma 5.3.3, $w_{1}$ has the prefix $0^{2^{k}} 1$, therefore $w_{0}>w_{1}$. Let $i \geq 1$. Property 5.3.1 implies that

$$
\Psi(\langle 1,0\rangle)>\Psi(\langle\lambda, 0\rangle)
$$

therefore

$$
\langle 1,0\rangle>\langle\lambda, 0\rangle
$$

and

$$
\langle 1,0\rangle>\varphi^{2}(\langle 1,0\rangle)
$$

since the first symbol of $\varphi^{2}(\langle 1,0\rangle)$ is $\langle\lambda, 0\rangle$. Corollary 3.2 .5 and Lemma 5.3.3 imply

$$
w_{i}=0 \Psi\left(\varphi^{2 i}(\langle 1,0\rangle) \varphi^{2 i+1}(\langle 1,0\rangle)\right) 0^{-1}>0 \Psi\left(\varphi^{2 i+2}(\langle 1,0\rangle) \varphi^{2 i+3}(\langle 1,0\rangle)\right) 0^{-1}=w_{i+1} .
$$

\section{Part C}

Lemma 5.3.7 For $i \geq 2,0^{2^{k}}$ is a prefix of $w_{i}$.

Proof. An easy application of Theorem 2.3.2.

Lemma 5.3.8 For $i \geq 1, w_{i}$ is a primitive word.

Proof. Denote $\omega=0^{-1} w_{1} 0=\Psi\left(\varphi^{2 i}(\langle 1,0\rangle) \varphi^{2 i+1}(\langle 1,0\rangle)\right)=\Psi\left(\varphi^{2 i-1}(\langle\lambda, 0\rangle a\langle\lambda, 0\rangle\langle 1,0\rangle b c)\right)$

$$
\text { where } \begin{cases}a=\langle 1,1\rangle, b=\langle\lambda, 1\rangle, c=\langle 1,0\rangle & \text { if } k=2 ; \\ a=\langle 11,0\rangle, b=\langle\lambda, 0\rangle, c=\langle 11,1\rangle & \text { if } k=3 ; \\ a=\langle 11,0\rangle, b=\langle\lambda, 0\rangle, c=\langle 111,0\rangle & \text { if } k>3 .\end{cases}
$$

If $w_{i}$ is not primitive then neither is $\omega$. The length of $\omega$ is $3 \cdot 2^{2 i}$, therefore $\omega$ is a concatenation of either two or three identical factors. Since both $\Psi$ and $\varphi$ are strictly growing, this implies $\langle\lambda, 0\rangle=\langle 1,0\rangle$ in the former case and $a=\langle 1,0\rangle$ in the latter case.

Lemma 5.3.9 For $i \geq 1, w_{i}$ is a Lyndon word.

$w_{0}$ is clearly a Lyndon word, since it is smaller than any of its non-empty proper suffixes. It is easy to check, that $w_{1}$ is of the form

$$
w_{1}=\left\{\begin{array}{cc}
0 \Psi(x\langle\lambda, 0\rangle\langle 1,0\rangle x\langle\lambda, 0\rangle\langle 1,1\rangle x\langle\lambda, 1\rangle\langle 1,1\rangle) 0^{-1}=0^{-1} 0^{4} 10^{3} 10^{3} 10^{2} 10^{4} 10^{2} 1 & \text { if } k=2 ; \\
0 \Psi\left(x\langle\lambda, 0\rangle\left\langle 1^{2}, 1\right\rangle x\langle\lambda, 0\rangle\left\langle 1^{2}, 0\right\rangle x\langle\lambda, 1\rangle\left\langle 1^{2}, 1\right\rangle\right) 0^{-1}=0^{8} 10^{4} 1^{2} 010^{7} 10^{6} 10^{8} 1^{5} 0^{2} 1 & \text { if } k=3 ; \\
0 \Psi\left(x\langle\lambda, 0\rangle\left\langle 1^{3}, 0\right\rangle x\langle\lambda, 0\rangle\left\langle 1^{2}, 0\right\rangle x\langle\lambda, 0\rangle\left\langle 1^{3}, 1\right\rangle\right) 0^{-1}= & \\
0^{16} 10^{12} 1^{2} 010^{15} 10^{14} 10^{16} 10^{8} 1^{4} 0^{2} 1 & \text { if } k=4 ; \\
0 \Psi\left(x\langle\lambda, 0\rangle\left\langle 1^{3}, 0\right\rangle x\langle\lambda, 0\rangle\left\langle 1^{2}, 0\right\rangle x\langle\lambda, 0\rangle\left\langle 1^{4}, 0\right\rangle\right) 0^{-1}= & \\
0^{2^{k}} 10^{2^{k}-4} 1^{2} 010^{2^{k}-1} 10^{2^{k}-2} 10^{2^{k}} 10^{2^{k}-8} 1^{4} 0^{2} 1 & \text { if } k>4 .
\end{array}\right.
$$


In each case, $w_{i}$ is smaller than any of its non-empty proper suffix.

Let now $i \geq 2$. By Lemma 5.3.4,

$$
w_{i}=0 \Psi\left(\varphi^{2 i}(\langle 1,0\rangle) \varphi^{2 i+1}(\langle 1,0\rangle)\right) 0^{-1}=0 \Psi\left(\varphi^{2 i-1}\left(\langle\lambda, 0\rangle\langle\langle 1,0\rangle) \varphi^{2 i+1}(\langle 1,0\rangle)\right) 0^{-1},\right.
$$

therefore $0^{-1} w_{i}$ has the same prefix $0^{2^{k}-1} 1$ as $t_{w}$ and $w_{i}$ has the prefix $0^{2^{k}} 1$. Lemma 5.3 .8 implies that $w_{i}$ is a primitive word. The word $w_{i}$ is a conjugate of $\Psi\left(\varphi^{2 i}(\langle 1,0\rangle) \varphi^{2 i+1}(\langle 1,0\rangle)\right)$. Assume that $w_{i}$ is not minimal in its conjugacy class. Let $w_{i}=u v$ where $v u<w_{i}$ is the smallest conjugate of $w_{i}$. The minimality of $v u$ implies that the last symbol of $u$ is 1 . The word $v u$ is of the form $\xi_{0} x_{1} x_{2} y \xi_{0}^{\prime}, \xi_{0} \neq \lambda$, where $\xi_{0}^{\prime} \xi_{0}=\Psi\left(c_{0}\right), x_{1}=\Psi\left(c_{1}\right), x_{2}=\Psi\left(c_{2}\right)$ for some $c_{0}, c_{1}, c_{2} \in \Sigma$ and $\xi_{0}^{\prime} \xi_{0} x_{1} x_{2} y=\Psi\left(c_{0} c_{1} c_{2} z\right)$ for some conjugate $c_{0} c_{1} c_{2} z$ of $\varphi^{2 i}(\langle 1,0\rangle) \varphi^{2 i+1}(\langle 1,0\rangle)$. Since $0^{2^{k}}$ is a prefix of $w_{i}, 0^{2^{k}}$ must be a prefix of $v u$. Therefore $\xi_{0} x_{1} \in 0^{*}, x_{1}=0^{2^{k-1}}$ and, consequently, $c_{1}=\langle\lambda, 0\rangle$. Property 5.3.2 then implies that $c_{0} \neq$ $\langle\lambda, 0\rangle, c_{2} \neq\langle\lambda, 0\rangle$ and from Property 5.3.1 we obtain that $\Psi\left(c_{2}\right)$ does not have the prefix $0^{2^{k-1}}$. Hence $\Psi\left(c_{0}\right)$ has the suffix 10 , therefore $\xi_{0}=0$.

This further implies that $x_{2}=0^{2^{k-1}-1} 1$ and $c_{2}=\langle 1,0\rangle$. Now either $c_{0} c_{1} c_{2} z$ or $c_{1} c_{2} z c_{0}$ is of the form $\varphi\left(z^{\prime}\right)$ where $z^{\prime}$ is a conjugate of $\varphi^{2 i-1}(\langle 1,0\rangle) \varphi^{2 i}(\langle 1,0\rangle)$.

The former case is not possible, since, by Property 5.3.2, $c_{1}=\langle\lambda, 0\rangle$ can occur only at even positions.

If $z^{\prime}=a z^{\prime \prime}$ where $a \in \Sigma$ then $\varphi(a)=c_{1} c_{2}=\langle\lambda, 0\rangle\left\langle\langle 1,0\rangle\right.$, hence $a=\langle\lambda, 0\rangle$. Now either $a z^{\prime \prime}=\varphi\left(y^{\prime}\right)$ or $z^{\prime \prime} a=\varphi\left(y^{\prime}\right)$ for some $y^{\prime} \in \Sigma^{*}$ being a conjugate of $\varphi^{2 i-2}(\langle 1,0\rangle) \varphi^{2 i-1}(\langle 1,0\rangle)$. Considering Property 5.3.2 again, $a$ must appear in $\varphi\left(y^{\prime}\right)$ at an even position, thus the former case takes place and

$$
0^{-1} v u 0=x_{1} x_{2} y \xi_{0}^{\prime} \xi_{0}=\Psi\left(\varphi\left(z^{\prime}\right)\right)=\Psi\left(\varphi^{2}\left(y^{\prime}\right)\right) .
$$

Moreover, since $a=\langle\lambda, 0\rangle$, the first symbol of $\varphi^{k-1}\left(y^{\prime}\right)$ is $\langle\lambda, 0\rangle$, and the first symbol of $\Psi\left(y^{\prime}\right)$ is 0 . Since $v u<w_{i}$,

$$
\Psi\left(\varphi^{2}\left(y^{\prime}\right)\right)=0^{-1} v u 0<0^{-1} w_{i} 0=\Psi\left(\varphi^{2 i}(\langle 1,0\rangle) \varphi^{2 i+1}(\langle 1,0\rangle)\right) .
$$

Thus

$$
\begin{gathered}
\varphi^{2}\left(y^{\prime}\right)<\varphi^{2 i}(\langle 1,0\rangle) \varphi^{2 i+1}(\langle 1,0\rangle), \\
y^{\prime}<\varphi^{2 i-2}(\langle 1,0\rangle) \varphi^{2 i-1}(\langle 1,0\rangle)
\end{gathered}
$$

and

$$
0^{-1} \Psi\left(y^{\prime}\right) 0<0^{-1} \Psi\left(\varphi^{2 i-2}(\langle 1,0\rangle) \varphi^{2 i-1}(\langle 1,0\rangle)\right) 0=w_{i-1} .
$$

However, the left-hand side is a conjugate of the right-hand side and we have a contradiction to the inductive hypothesis that $w_{i-1}$ is a Lyndon word. This concludes the proof of Theorem 4.1.1

Theorem 4.3.1 follows from Lemma 5.3.5, Lemma 5.3.6, and Lemma 5.3.9.

\subsection{Case $w=10^{k-1}$}

Let $w=10^{k-1}, k \geq 2$. In this case $s=k-1, \Psi=\psi \circ \varphi^{2 k-2}$ and each element of $\Sigma$ is of the form $\langle\lambda, m\rangle$ or $\left\langle 10^{r}, m\right\rangle$ for some $r \in[k-1], m \in[2]$. The initial state of $\mathfrak{A}$ is $a_{0}=\langle\lambda, 0\rangle$.

The first of the next two properties may be observed applying Remark 2.1.2. The second follows from the fact that the only prefix of $w$, which ends in 1 is 1 . 


\section{Property 5.4.1}

(i) $\varphi(\langle\lambda, 0\rangle)=\langle\lambda, 0\rangle\langle 1,0\rangle, \varphi(\langle 1,0\rangle)=\langle\lambda, 1\rangle\langle 1,0\rangle$,

(ii) $\psi\left(\varphi^{k-1}(\langle\lambda, 0\rangle)\right)=0^{2^{k-1}}$ and, for $r \in[k-1], \psi\left(\varphi^{k-1}\left(\left\langle 10^{r}, 0\right\rangle\right)\right)=1^{2^{r}} 0^{2^{k-1}-2^{r}}$.

Property 5.4.2 If a symbol $a \in \Sigma$ occurs in an odd position of a word of the form $\varphi(x), x \in \Sigma^{*}$, then $a=\langle 1,0\rangle$ or $a=\langle 1,1\rangle$.

\section{Proof of Theorem 4.4.1}

\section{Part $A$}

\section{Lemma 5.4.3}

(i) $\mu^{k-1}\left(0^{2^{k-1}}\right)$ has the suffix $0^{2^{k-1}-1}$.

(ii) $\rho=\Psi\left(\varphi^{k-1}(\langle\lambda, 1\rangle)\right) \Psi(\langle\lambda, 0\rangle)\left(0^{2^{k-1}-1}\right)^{-1}$.

(iii) For $i \geq 0, \Psi\left(\varphi^{2 k-1+i}(\langle 1,0\rangle)\right)$ has the prefix $\rho 0^{2^{k}} 1$.

(iv) $w_{2 k-2}$ has the suffix $\rho$.

(v) For $i \geq 2 k-1, w_{i}=\rho^{-1} \Psi\left(\varphi^{i}(\langle 1,0\rangle)\right) \rho$ and $\mu\left(\rho w_{i} \rho^{-1}\right)$ has the prefix $\rho$.

\section{Proof.}

(i) Property 2.3 .3 implies

$$
\mu^{k-1}\left(0^{2^{k-1}}\right)=\mu^{k-1}\left(\psi\left(\varphi^{k-1}(\langle\lambda, 0\rangle)\right)\right)=\psi\left(\varphi^{2 k-2}(\langle\lambda, 0\rangle)\right)=\psi\left(\varphi^{k-1}\left(\varphi^{k-1}(\langle\lambda, 0\rangle)\right)\right) .
$$

Using Remark 2.1.2 it is easy to observe that the last symbol of $\varphi^{k-1}(\langle\lambda, 0\rangle)$ is $\langle 1,0\rangle$. Hence $\mu^{k-1}\left(0^{2^{k-1}}\right)$ has the suffix $\psi\left(\varphi^{k-1}(\langle 1,0\rangle)\right)=10^{2^{k-1}}$.

(ii) Again applying Property 2.3.3 we obtain $\mu^{2 k-2}\left(1^{2^{k-1}}\right)=\Psi\left(\varphi^{k-1}(\langle\lambda, 0\rangle)\right)$ and $\mu^{k-1}\left(0^{2^{k-1}}\right)=\Psi(\langle\lambda, 0\rangle)$ and, consequently, $\rho=\Psi\left(\varphi^{k-1}(\langle\lambda, 1\rangle)\right) \Psi(\langle\lambda, 0\rangle)\left(0^{2^{k-1}-1}\right)^{-1}$.

(iii) Let $i \geq 0$. Denote $v=\Psi\left(\varphi^{2 k-1+i}(\langle 1,0\rangle)\right)=\Psi\left(\varphi^{k-1}\left(\varphi^{i}\left(\varphi\left(\varphi^{k-1}(\langle 1,0\rangle)\right)\right)\right)\right)$. The word $\varphi^{k-1}(\langle 1,0\rangle)$ has the prefix $\langle\lambda, 1\rangle$ therefore $v$ has the prefix

$$
\Psi\left(\varphi^{k-1}\left(\varphi^{i}(\varphi(\langle\lambda, 1\rangle))\right)\right)=\Psi\left(\varphi^{k-1}\left(\varphi^{i}(\langle\lambda, 1\rangle\langle 1,1\rangle)\right)\right)
$$

Now either

$$
i=0 \text { and } \varphi^{i}(\langle\lambda, 1\rangle\langle 1,1\rangle)=\langle\lambda, 1\rangle\langle 1,1\rangle
$$

or

$$
i>0 \text { and } \varphi^{i}(\langle\lambda, 1\rangle) \text { has the prefix }\langle\lambda, 1\rangle\langle 1,1\rangle,
$$

in either case $v$ has the prefix $=\Psi\left(\varphi^{k-1}(\langle\lambda, 1\rangle)\right) \Psi\left(\varphi^{k-1}(\langle 1,1\rangle)\right)$. Thus $v$ has the prefix

$$
\Psi\left(\varphi^{k-1}(\langle\lambda, 1\rangle)\right) \Psi(\langle\lambda, 0\rangle\langle 1,1\rangle)
$$

having the prefix $\left(\rho 0^{2^{k-1}-1}\right) 0^{2^{k-1}+1} 1$, since $\varphi^{k-1}(\langle 1,1\rangle)$ starts by $\langle\lambda, 0\rangle\langle 1,1\rangle$. 
(iv) $w_{2 k-2}$ is the factor of $t_{w}$ of length $j_{2 k-1}-j_{2 k-2}$ at position $j_{2 k-2}$. The length of $\rho$ is

$$
2^{3 k-3}+2^{2 k-2}-2^{k-1}+1
$$

The suffix of $w_{2 k-2}$ of length $|\rho|$ is a factor of $t_{w}$ at position

$$
j_{2 k-2}+\left|w_{2 k-1}\right|-|\rho|=j_{2 k-1}-|\rho|=2^{4 k-3} .
$$

This is the starting position of the factor $\Psi\left(\varphi^{2 k-1}(\langle 1,0\rangle)\right)$, which by (iii) has the prefix $\rho$.

(v) We will prove the assertion by induction. Let first $i=2 k-1$. The factor of length $|\rho|$ preceding the factor $w_{2 k-1}$ in $t_{w}$ starts at position $j_{2 k-1}-|\rho|=2^{4 k-3}$. This is the starting position of the factor $\Psi\left(\varphi^{2 k-1}(\langle 1,0\rangle)\right)$, which by (iii) has the prefix $\rho$. Thus the first part of the assertion has been proved in (111).

Since $\left|w_{2 k-1}\right|=j_{2 k-1}-|\rho|=2^{4 k-3}$, the word $\rho w_{2 k-1} \rho^{-1}$ is identical with $\Psi\left(\varphi^{2 k-1}(\langle 1,0\rangle)\right)$ and $w_{2 k-1}=\rho^{-1} \Psi\left(\varphi^{2 k-1}(\langle 1,0\rangle)\right) \rho$. Now

$$
\mu\left(\rho w_{2 k-1} \rho^{-1}\right)=\mu\left(\Psi\left(\varphi^{2 k-1}(\langle 1,0\rangle)\right)\right)=\Psi\left(\varphi^{2 k}(\langle 1,0\rangle)\right)
$$

having the prefix $\rho$ by (iii).

Assume now that the assertion is true for some $i \geq 2 k-1$. Then

$$
w_{i+1}=\rho^{-1} \mu\left(\rho w_{i} \rho^{-1}\right) \rho=\rho^{-1} \mu\left(\Psi\left(\varphi^{i}(\langle 1,0\rangle)\right)\right) \rho=\rho^{-1} \Psi\left(\varphi^{i+1}(\langle 1,0\rangle)\right) \rho
$$

and

$$
\mu\left(\rho w_{i+1} \rho^{-1}\right)=\mu\left(\Psi\left(\varphi^{i+1}(\langle 1,0\rangle)\right)\right)=\Psi\left(\varphi^{i+2}(\langle 1,0\rangle)\right)
$$

has the prefix $\rho$ according to (iii).

Lemma 5.4.4 $t_{w}=\prod_{i=0}^{\infty} w_{i}$

Proof. Since $t_{w}$ is a fixed point of $\mu$ with the prefix $w_{0}$ of length greater than $2^{k-1}$, it is enough to prove that the sequence $\prod_{i=0}^{\infty} w_{i}$ having the same prefix is a fixed point of $\mu$ as well. From the definition of the words $w_{0}, w_{2 k-1}, \rho$ we can observe that $w_{0} w_{1} \cdots w_{2 k-2} w_{2 k-1}$ is a prefix of $t_{w}$, the length of $w_{0} w_{1} \cdots w_{2 k-2} \rho^{-1}$ is twice the length of $w_{0} w_{1} \cdots w_{2 k-2} \rho^{-1}$ and the length of the latter word is a multiple of $2^{k-1}$. We obtain:

$$
\begin{aligned}
\mu\left(\prod_{i=0}^{\infty} w_{i}\right) & \left.=\mu\left(w_{0} w_{1} \cdots\left(w_{2 k-2} \rho^{-1}\right) \rho w_{2 k-1} \rho^{-1}\right) \prod_{i=2 k}^{\infty}\left(\rho w_{i} \rho^{-1}\right)\right) \\
& =\mu\left(w_{0} w_{1} \cdots w_{2 k-2} \rho^{-1}\right) \mu\left(\rho w_{2 k-1} \rho^{-1}\right) \prod_{i=2 k}^{\infty} \mu\left(\rho w_{i} \rho^{-1}\right) \\
& =\left(w_{0} w_{1} \cdots w_{2 k-2} w_{2 k-1} \rho^{-1}\right) \rho w_{2 k} \rho^{-1} \prod_{i=2 k}^{\infty}\left(\rho w_{i+1} \rho^{-1}\right) \\
& =\prod_{i=0}^{\infty} w_{i} .
\end{aligned}
$$

since $\left|w_{0} w_{1} \cdots\left(w_{2 k-2} \rho^{-1}\right)\right|=2^{4 k-3}$ and $\left|w_{0} w_{1} \cdots w_{2 k-2}\left(w_{2 k-1} \rho^{-1}\right)\right|=2^{4 k-2}$. 


\section{Part $B$}

Lemma 5.4.5 For $0 \leq i \leq 2 k-1$ the last symbol in $w_{i}$ is 1 .

Proof. The last symbol of $w_{i}$ occurs at position $j_{i+1}-1$ in $t_{w}$. The binary representation of each $j_{i+1}-1$ contains an odd number of occurrences of $w$.

\section{Lemma 5.4.6}

$w_{0}$ has the prefix $0^{2^{k-1}} 1$,

$w_{1}, w_{2}$, have the prefix $0^{2^{k-1}+1} 1$, for $1 \leq r \leq k-2$,

$w_{2 r+1}$ and $w_{2 r+2}$ have the prefix $0^{2^{k-1}-1+2^{r}} 1$ and

$w_{2 k-1}$ has the prefix $0^{2^{k}} 1$.

Proof. A simple observation of the shape of binary representation of the position numbers following $j_{i}$, $0 \leq i \leq 2 k$.

Lemma 5.4.7 For $0 \leq i \leq 2 k-2, w_{i}>w_{i+1}$.

Proof. Lemma 5.4.6 implies that $w_{0}>w_{1}$ and, for $2 \leq r \leq k-1, w_{2 r}>w_{2 r+1}$.

Let $0 \leq r \leq k-2$. The word $w_{2 r+1}$ occurs as a factor of length $2^{3 k-2+r}$ in $t_{w}$ at position

$$
j_{2 r+1}=2^{3 k-2+r}+2^{2 k-2+r}+2^{k-1+r}-2^{k-1}+d
$$

where $d=0$ if $r=0$ and $d=1$ otherwise.

Therefore the suffix of

$$
v=\psi\left(\varphi^{3 k-2+r}(\langle 1,0\rangle)\right)=\Psi\left(\varphi^{r}\left(\varphi^{k}(\langle 1,0\rangle)\right)\right.
$$

at position

$$
2^{2 k-2+r}+2^{k-1+r}-2^{k-1}+1
$$

is a prefix of $w_{2 r+1}$.

Since $\varphi^{k}(\langle 1,0\rangle)$ starts by $\langle\lambda, 1\rangle\langle 1,1\rangle b$ where $b=\langle 10,0\rangle$ if $k \geq 3$ and $b=\langle\lambda, 1\rangle$ if $k=2$, the suffix of $\Psi\left(\varphi^{r}(\langle 1,1\rangle b)\right)$ at position $2^{k-1+r}-2^{k-1}+d$ is a prefix of $w_{2 r+1}$.

In a similar way, the suffix of

$$
v=\psi\left(\varphi^{3 k-2+r+1}(\langle 1,0\rangle)\right)=\Psi\left(\varphi^{r}\left(\varphi^{k+1}(\langle 1,0\rangle)\right)\right.
$$

at position $2^{2 k-2+r}+2^{k-1+r}-2^{k-1}+d$ is a prefix of $w_{2 r+2}$.

Since $\varphi^{k+1}(\langle 1,0\rangle)$ starts by $\langle\lambda, 1\rangle\langle 1,1\rangle c$ where $c=\langle 10,1\rangle$ if $k \geq 3$ and $c=\langle\lambda, 0\rangle$ if $k=2$, the suffix of $\Psi\left(\varphi^{r}(\langle 1,1\rangle c)\right)$ at position $2^{k-1+r}-2^{k-1}+d$ is a prefix of $w_{2 r+2}$. In any case, $\Psi(b)$ starts by 1 and $\Psi(c)$ starts by 0 , hence $w_{2 r+1}>w_{2 r+2}$.

We still have to prove that $w_{2}>w_{3}$ for $k \geq 3$.

Since $\varphi^{k-1}(\langle 1,1\rangle)$ has the prefix $\langle\lambda, 0\rangle\langle 1,1\rangle\langle 10,1\rangle\langle 1,1\rangle$, the suffix of $\Psi(\langle 1,1\rangle\langle 10,1\rangle)$ at position $2^{k-1+0}-2^{k-1}+0=0$ has the prefix

$$
\left(0^{2^{k-1}}\right)\left(01^{2^{k-1}-1}\right)\left(0^{2} 1^{2^{k-1}-2}\right)\left(01^{2^{k-1}-1}\right)
$$


and $w_{2}$ has the prefix $0^{2^{k-1}+1} 1^{2^{k-1}-1}$.

The word $\varphi(\langle 1,1\rangle\langle 10,0\rangle)$ has the prefix $\langle 10,1\rangle\langle 1,1\rangle$ and $\varphi^{k-1}(\langle 10,1\rangle\langle 1,1\rangle)$ has the prefix

$$
\langle\lambda, 0\rangle\langle 1,0\rangle\langle 10,1\rangle\langle 1,1\rangle \text {. }
$$

Therefore the suffix of

$$
\Psi(\varphi(\langle 1,1\rangle\langle 10,0\rangle))
$$

at position

$$
2^{k-1+1}-2^{k-1}+1=2^{k-1}+1
$$

has the prefix

$$
\left(0^{2^{k-1}}\right)\left(10^{2^{k-1}-1}\right)\left(0^{2} 1^{2^{k-1}-2}\right)\left(01^{2^{k-1}-1}\right)
$$

and $w_{3}$ has the prefix

$$
0^{2^{k-1}+1} 1^{2^{k-1}-2} 01^{2^{k-1}-1}
$$

Clearly, $w_{2}>w_{3}$.

Lemma 5.4.8 For $i \geq 2 k-1, w_{i}>w_{i+1}$

Proof. Using Remark 2.1.2, it is not difficult to observe that $\Psi(\langle 1,0\rangle)$ has the prefix $1^{2^{k-1}+1}$ while $\Psi(\langle\lambda, 1\rangle)$ has the prefix $1^{2^{k-1}} 0$. Hence $\Psi(\langle 1,0\rangle)>\Psi(\langle\lambda, 1\rangle)$ and $\langle 1,0\rangle>\langle\lambda, 1\rangle$. Property 5.4.1 implies that $\langle 1,0\rangle>\varphi(\langle 1,0\rangle)$ and $\mathrm{V}$ of Lemma 5.4 .3 implies $w_{i}>w_{i+1}$.

\section{Part C}

Lemma 5.4.9 Let $\tau=\lim _{n \rightarrow \infty} \varphi^{n}(\langle\lambda, 0\rangle)$.

(i) The first two occurrences of the factor $\langle\lambda, 0\rangle$ in $\tau$ are at positions $\left\lfloor j_{0} / 2^{k-1}\right\rfloor$ and $\left\lfloor j_{1} / 2^{k-1}\right\rfloor$.

(ii) The first two occurrences of the factor $\langle\lambda, 0\rangle\langle 1,1\rangle$ in $\tau$ are at positions $\left\lfloor j_{1} / 2^{k-1}\right\rfloor$ and $\left\lfloor j_{2} / 2^{k-1}\right\rfloor$.

(iii) For $1 \leq r \leq k-2$, the first two occurrences of the factor $\langle 1,0\rangle\left\langle 10^{r}, 1\right\rangle$ are at positions $\left\lfloor j_{2 r+1} / 2^{k-1}\right\rfloor$ and $\left\lfloor j_{2 r+2} / 2^{k-1}\right\rfloor$.

(iv) The first two occurrences of the factor $\langle 1,0\rangle\langle\lambda, 0\rangle\langle 1,1\rangle$ in $\tau$ are at positions $\left\lfloor j_{2 k-1} / 2^{k-1}\right\rfloor$ and $\left\lfloor j_{2 k} / 2^{k-1}\right\rfloor$.

(v) The first occurrence of the factor $\langle 1,0\rangle\langle\lambda, 0\rangle\langle 1,0\rangle$ in $\tau$ is at a position greater than $\left\lfloor j_{2 k-1} / 2^{k-1}\right\rfloor$.

\section{Proof.}

(i) The first occurrence of $\langle\lambda, 0\rangle$ after position $0=\left\lfloor j_{0} / 2^{k-1}\right\rfloor$ is at the position $\left(10^{k-1} 10^{k-1}\right)_{[2]}=\left\lfloor j_{1} / 2^{k-1}\right\rfloor$.

(ii) If the factor $\langle\lambda, 0\rangle\langle 1,1\rangle$ occurs in $\tau$ at (an even) position $j$ then the symbol at position $j / 2$ is $\left\langle 10^{k-2}, 1\right\rangle$. This must be a prefix of a factor $\varphi^{k-2}(\langle 1,1\rangle)$. Since the first two occurrences of $\langle 1,1\rangle$ in $\tau$ are at positions $\left(10^{k-1} 1\right)_{[2]}=2^{k}+1$ and $\left(10^{k} 1\right)_{[2]}=2^{k+1}+1$, the first two occurrences of the factor $\langle\lambda, 0\rangle\langle 1,1\rangle$ in $\tau$ are at positions $\left(2^{k}+1\right) \cdot 2^{k-2} \cdot 2=2^{2 k-1}+2^{k-1}=\left\lfloor j_{1} / 2^{k-1}\right\rfloor$ and $\left(2^{k+1}+1\right) \cdot 2^{k-2} \cdot 2=2^{2 k}+2^{k-1}=\left\lfloor j_{2} / 2^{k-1}\right\rfloor$. 
(iii) If the factor $\langle 1,0\rangle\left\langle 10^{r}, 1\right\rangle$ occurs in $\tau$ then $\left\langle 10^{r}, 1\right\rangle$ is the prefix of a factor $\varphi^{r}(\langle 1,1\rangle)$. In that case $\langle 1,0\rangle$ is a suffix of some $\varphi^{r}(b), b \in \Sigma$. Then $b=\langle\alpha, 0\rangle$ where $\alpha \neq 1$, since $b\langle 1,1\rangle$ is a factor of $\tau$ and $\langle 1,1\rangle$ must be in an odd position. Then $b\langle 1,1\rangle=\varphi(c)$ for some $c \in \Sigma$. This is only possible if $c=\left\langle 10^{k-2}, 1\right\rangle$ and $c$ is a prefix of a factor $\varphi^{k-2}(\langle 1,1\rangle)$. Since the first two occurrences of $\langle 1,1\rangle$ in $\tau$ are at positions $\left(10^{k-1} 1\right)_{[2]}=2^{k}+1$ and $\left(10^{k} 1\right)_{[2]}=2^{k+1}+1$, the first two occurrences of the factor $\langle 1,0\rangle\left\langle 10^{r}, 1\right\rangle$ in $\tau$ are at positions

$$
\left(2^{k}+1\right) \cdot 2^{k-2} \cdot 2 \cdot 2^{r}+2^{r}-1=2^{2 k-1+r}+2^{k-1+r}+2^{r}-1=\left\lfloor j_{2 r+1} / 2^{k-1}\right\rfloor
$$

and

$$
\left(2^{k+1}+1\right) \cdot 2^{k-2} \cdot 2 \cdot 2^{r}+2^{r}-1=2^{2 k+r}+2^{k-1+r}+2^{r}-1=\left\lfloor j_{2 r+2} / 2^{k-1}\right\rfloor .
$$

(iv) Let the factor $\langle 1,0\rangle\langle\lambda, 0\rangle\langle 1,1\rangle$ occur in $\tau$ at (an odd) position $j$. Then the factor of length 2 at position $\lfloor j / 2\rfloor$ is $\langle 1,0\rangle\left\langle 10^{k-2}, 1\right\rangle$ and in the same way (taking $r=k-2$ ) as in the proof of iii we deduce that $\lfloor j / 2\rfloor$ corresponds to the position $2\left(2^{k-2}-1\right)+1$ of a factor $\varphi^{1+(k-2)+1+(k-2)}(\langle 1,1\rangle)$, hence the first two occurrences of the factor $\langle 1,0\rangle\langle\lambda, 0\rangle\langle 1,1\rangle$ in $\tau$ are at positions

$$
\left(2^{k}+1\right) \cdot 2 \cdot 2^{k-2} \cdot 2 \cdot 2^{k-2}+2^{k-1}-1=2^{3 k-2}+2^{2 k-2}+2^{k-1}-1=\left\lfloor j_{2 k+1} / 2^{k-1}\right\rfloor
$$

and

$$
\left(2^{k+1}+1\right) \cdot 2 \cdot 2^{k-2} \cdot 2 \cdot 2^{k-2}+2^{k-1}-1=2^{3 k-1}+2^{2 k-2}+2^{k-1}-1=\left\lfloor j_{2 k+2} / 2^{k-1}\right\rfloor .
$$

(v) Let the factor $\langle 1,0\rangle\langle\lambda, 0\rangle\langle 1,0\rangle$ occur in $\tau$ for the first time at (an odd) position $j$. Then the factor of length 3 at position $\lfloor j / 2\rfloor$ is either $\langle 1,0\rangle\langle\lambda, 0\rangle\langle 1,0\rangle$ or $\langle 1,0\rangle\langle\lambda, 0\rangle\langle 1,1\rangle$. The former case would mean an earlier occurrence of the factor, hence the latter case take place. The factor $\langle 1,0\rangle\langle\lambda, 0\rangle\langle 1,0\rangle$ hence occurs later than $\langle 1,0\rangle\langle\lambda, 0\rangle\langle 1,1\rangle$.

Lemma 5.4.10 For $0 \leq i \leq 2 k-1, w_{i}$ is a Lyndon word.

Proof. The binary representation of each $j_{i+1}-1$ contains an odd number of occurrences of $10^{k-1}$, and therefore the last symbol of each $w_{i}$ is 1 . To prove that $w_{i}$ is a Lyndon word, we will show that $w_{i}$ besides in its prefix $0^{p} 1$ (as described by Lemma 5.4.6), does not contain any occurrence of the factor $0^{p}$. We will use Lemma 5.4.9, which will imply in each particular case that the next occurrence of $0^{p}$ in $t$, after the prefix of $w_{i}$, is in some of the following factors $w_{j}$.

1. $i=0$. Property 5.4 .1 implies that a factor $0^{2^{k-1}}$ occurs in $t_{w}$ at some position $j$ only if either $\langle\lambda, 0\rangle$ or, for some $r \geq s \geq 0,\left\langle 10^{s}, 0\right\rangle\left\langle 10^{r}, 1\right\rangle$ occurs at position $\left\lfloor j / 2^{k-1}\right\rfloor$ of $\tau=\lim _{n \rightarrow \infty} \varphi^{n}(\langle\lambda, 0\rangle)$. Property 5.4.2 implies that in the latter case exactly one of $r, s$ must be equal to 0 , hence $r>s=0$ and the factor has the form $\langle 1,0\rangle\left\langle 10^{r}, 1\right\rangle$ for some $r \geq 1$. We apply 1 and iii of Lemma 5.4.9.

2. $i=1$ or $i=2$. A factor $0^{2^{k-1}+1}$ occurs in $t_{w}$ at position $j$ only if either $\langle\lambda, 0\rangle\langle 1,1\rangle$ or $\langle 1,0\rangle\left\langle 10^{r}, 1\right\rangle$, for some $r \geq 1$, or $\langle 1,0\rangle\langle\lambda, 0\rangle$ occurs at position $\left\lfloor j / 2^{k-1}\right\rfloor$ of $\tau$. We apply ii and iii of Lemma 5.4.9. 
3. $i=3$ or $i=4, k \geq 3$. As shown in the proof of Lemma 5.4.7, $w_{3}$ has the prefix $0^{2^{k-1}+1} 1^{2^{k-1}-2} 01^{2^{k-1}-1}$ and $w_{4}$ the same or smaller prefix. If a factor $0^{2^{k-1}+1}$ occurs in $w_{3}$ or $w_{4}$ at the position $j$ of $t_{w}$ then the factor $\langle\lambda, 0\rangle\langle 1,1\rangle$ occurs at position $\left\lfloor j / 2^{k-1}\right\rfloor$ of $\tau$. Hence $0^{2^{k-1}+1}$ occurs in $w_{3}$ or $w_{4}$ as a prefix of the factor $0^{2^{k-1}} 01^{2^{k-1}-1}>02^{2^{k-1}+1} 1^{2^{k-1}-2} 01^{2^{k-1}-1}$.

4. $i=2 r+1$ or $i=2 r+2,2 \leq r \leq k-2$. A factor $0^{2^{k-1}-1+2^{r}}$, occurs in $t_{w}$ at position $j$ only if either $\langle 1,0\rangle\langle\lambda, 0\rangle$ or $\langle 1,0\rangle\left\langle 10^{r}, 1\right\rangle$ occurs at position $\left\lfloor j / 2^{k-1}\right\rfloor$ of $\tau$. If the factor $\langle 1,0\rangle\langle\lambda, 0\rangle$ occurs in $\tau$, then it is a prefix of the factor $\langle 1,0\rangle\langle\lambda, 0\rangle\langle 1,1\rangle$ or $\langle 1,0\rangle\langle\lambda, 0\rangle\langle 1,0\rangle$. We apply iiil and iv and $\nabla$ of Lemma 5.4.9.

5. $i=2 k-1$. A factor $0^{2^{k}}$ occurs in $t_{w}$ at position $j$ only if $\langle 1,0\rangle\langle\lambda, 0\rangle\langle 1,1\rangle$ occurs at position $\left\lfloor j / 2^{k-1}\right\rfloor$ of $\tau$. We apply $i v$ of Lemma 5.4.9.

Lemma 5.4.11 For $i \geq 2 k-1, w_{1}$ is a primitive word.

Proof. Analogous to the proof of Lemma 5.1.13.

Lemma 5.4.12 For $i \geq 2 k-1, w_{i}$ is a Lyndon word.

Proof. We proceed by induction. The assertion for $i=2 k-1$ is implied by Lemma 5.4.10.

Let the assertion be true for some $i-1 \geq 2 k-1$. Lemma 5.4.11 implies that $w_{i}$ is a primitive word. By Lemma 5.4.3, $w_{i}$ is a conjugate of $\Psi\left(\varphi^{i}(\langle 1,0\rangle)\right)$. Assume that $w_{i}$ is not minimal in its conjugacy class. Let $w_{i}=u v$ where $v u<w_{i}$ is the smallest conjugate of $w_{i}$. According to Lemma 5.4.8, $w_{i}<w_{2 k-1}$, hence $w_{i}$, and $v u$ have the same prefix $0^{2^{k}} 1$ as $w_{2 k-1}$ because no conjugate of $\Psi\left(\varphi^{i}(\langle 1,0\rangle)\right)$ may contain the factor $0^{2^{k}+1}$, as one easily observes using Property 5.4.1. The word $v u$ is of the form $\xi_{0} x_{1} x_{2} x \xi_{0}^{\prime}$, $\xi_{0} \neq \lambda$, where $\xi_{0}^{\prime} \xi_{0}=\psi\left(\varphi^{k-1}\left(c_{0}\right)\right), x_{1}=\psi\left(\varphi^{k-1}\left(c_{1}\right)\right), x_{2}=\psi\left(\varphi^{k-1}\left(c_{2}\right)\right)$ for some $c_{0}, c_{1}, c_{2} \in \Sigma$ and $\xi_{0}^{\prime} \xi_{0} x_{1} x_{2} x=\psi\left(\varphi^{k-1}\left(c_{1} c_{2} x^{\prime} c_{0}\right)\right.$ for some conjugate $c_{1} c_{2} x^{\prime} c_{0}$ of $\varphi^{k-1+i}(\langle 1,0\rangle)$.

Property 5.4.1 in combination with Property 5.4.2 implies that the only possibility is $\xi_{0}=0^{2^{k-1}-1}$, $\xi_{0}^{\prime}=1, x_{1}=0^{2^{k-1}}, x_{2}=01^{2^{k-1}-1}, c_{0}=\langle 1,0\rangle, c_{1}=\langle\lambda, 0\rangle$ and $c_{2}=\langle 1,1\rangle$.

A repeated application of Property 5.4.2 leads to the conclusion that $c_{1} c_{2} x^{\prime} c_{0}$ is a conjugate of

$$
\varphi^{k-1+i}(\langle 1,0\rangle) \quad \text { of the form } \varphi^{k-1}(\langle 1,1\rangle y\langle\alpha, 0\rangle),
$$

where $\langle 1,1\rangle y\langle\alpha, 0\rangle$ is a conjugate of $\varphi^{i}(\langle 1,0\rangle)$, since $c_{1} c_{2}$ may occur only as a prefix of $\varphi^{k-1}(\langle 1,1\rangle)$.

Hence $\xi_{0}^{-1} v u \xi_{0}=x_{1} x_{2} z \xi_{0}^{\prime} \xi_{0}=\Psi(\langle 1,1\rangle y\langle\alpha, 0\rangle)$ for a conjugate $\langle 1,1\rangle y\langle\alpha, 0\rangle$ of $\varphi^{i}(\langle 1,0\rangle)$. Property 5.4 .2 now implies that $\langle\alpha, 0\rangle\langle 1,1\rangle y=\varphi\left(y^{\prime}\right)$ for some conjugate $y^{\prime}$ of $\varphi^{i-1}(\langle 1,0\rangle)$.

Again, the only possibility is that $\alpha=\lambda$ and $\langle\lambda, 0\rangle\langle 1,1\rangle y=\varphi^{k-1}\left(\langle 1,1\rangle b y^{\prime \prime}\right)$ for some conjugate $\langle 1,1\rangle b y^{\prime \prime}$ of $\varphi^{i-(k-1)}(\langle 1,0\rangle)$ where $b$ is some symbol from $\Sigma$ occurring at an even position and therefore different from $\langle 1,0\rangle$ and $\langle 1,1\rangle$. We will use the fact that, since $b \neq\langle 1,0\rangle$ and $b \neq\langle 1,1\rangle, \varphi^{k-2}(b)$ starts by $c \in \Sigma$ where either $c=\langle\lambda, 0\rangle$ or $c=\langle\lambda, 1\rangle$. Hence $y^{\prime}=\varphi^{k-2}(\langle 1,1\rangle) c z$ for some $z \in \Sigma^{*}$.

We have $\langle\lambda, 0\rangle\langle 1,1\rangle y=\varphi^{k-1}(\langle 1,1\rangle) \varphi(c z)$, where either $c=\langle\lambda, 0\rangle$ or $c=\langle\lambda, 1\rangle$ and $\varphi^{k-2}(\langle 1,1\rangle) c z$ is a conjugate of $\varphi^{i-1}(\langle 1,0\rangle)$. 
Furthermore, $\xi_{0}^{-1} v u \xi_{0}=\Psi(\langle 1,1\rangle y\langle\lambda, 0\rangle)$ and, finally, $\rho=\Psi\left(\varphi^{k-1}(\langle\lambda, 1\rangle)\langle\lambda, 0\rangle\right) \xi_{0}^{-1}$ as stated by iii of Lemma 5.4.3. We get

$$
\begin{aligned}
v u & <w_{i}=\rho^{-1} \Psi\left(\varphi^{i}(\langle 1,0\rangle)\right) \rho \\
\rho v u & <\Psi\left(\varphi^{i}(\langle 1,0\rangle)\right) \rho \\
\left(\rho \xi_{0}\right)\left(\xi_{0}^{-1} v u \xi_{0}\right) & <\Psi\left(\varphi^{i}(\langle 1,0\rangle)\right) \rho \xi_{0} \\
\Psi\left(\varphi^{k-1}(\langle\lambda, 1\rangle)\langle\lambda, 0\rangle\right) \Psi(\langle 1,1\rangle y\langle\lambda, 0\rangle) & \left.<\Psi\left(\varphi^{i}(\langle 1,0\rangle)\right)\right) \Psi\left(\varphi^{k-1}(\langle\lambda, 1\rangle)\langle\lambda, 0\rangle\right) \\
\varphi^{k-1}(\langle\lambda, 1\rangle)\langle\lambda, 0\rangle\langle 1,1\rangle y\langle\lambda, 0\rangle & <\varphi^{i}(\langle 1,0\rangle) \varphi^{k-1}(\langle\lambda, 1\rangle)\langle\lambda, 0\rangle \\
\varphi^{k-1}(\langle\lambda, 1\rangle) \varphi^{k-1}(\langle 1,1\rangle) \varphi(c z) & <\varphi^{i}(\langle 1,0\rangle) \varphi^{k-1}(\langle\lambda, 1\rangle) \\
\varphi^{k-2}\left(\langle\lambda, 1\rangle \varphi^{k-2}(\langle 1,1\rangle) c z\right. & <\varphi^{i-1}(\langle 1,0\rangle) \varphi^{k-2}(\langle\lambda, 1\rangle) \\
\varphi^{k-2}(\langle\lambda, 1\rangle\langle 1,1\rangle) c z & <\varphi^{i-1}(\langle 1,0\rangle) \varphi^{k-2}(\langle\lambda, 1\rangle) \\
\varphi^{k-1}(\langle\lambda, 1\rangle) c z & <\varphi^{i-1}(\langle 1,0\rangle) \varphi^{k-2}(\langle\lambda, 1\rangle) \\
\varphi^{k-1}(\langle\lambda, 1\rangle) c z & <\varphi^{i-1}(\langle 1,0\rangle) \varphi^{k-2}(\langle\lambda, 1\rangle) \\
\Psi\left(\varphi^{k-1}(\langle\lambda, 1\rangle) c z\right) & <\Psi\left(\varphi^{i-1}(\langle 1,0\rangle) \varphi^{k-2}(\langle\lambda, 1\rangle)\right)
\end{aligned}
$$

The word $\Psi\left(\varphi^{i-1}(\langle 1,0\rangle)\right)=\rho w_{i-1} \rho^{-1}$ has the prefix $\rho=\Psi\left(\varphi^{k-1}(\langle\lambda, 1\rangle)\right) \Psi\left(\langle\lambda, 0\rangle \xi_{0}^{-1}\right.$. The latter inequality therefore implies that the first symbol of $\Psi(c)$ is not greater than 0 being the first symbol of $\Psi(\langle\lambda, 0\rangle) \xi_{0}^{-1}$. The word $\Psi(\langle\lambda, 0\rangle)$ starts by 0 and $\Psi(\langle\lambda, 1\rangle)$ starts by 1 , hence $c=\langle\lambda, 0\rangle$. Then

$$
\begin{aligned}
\Psi\left(\varphi^{k-1}(\langle\lambda, 1\rangle)\langle\lambda, 0\rangle z\right) & <\Psi\left(\varphi^{i-1}(\langle 1,0\rangle) \varphi^{k-2}(\langle\lambda, 1\rangle)\right) \\
\Psi\left(\varphi^{k-1}(\langle\lambda, 1\rangle)\right) \Psi(\langle\lambda, 0\rangle) \Psi(z) & <\Psi\left(\varphi^{i-1}(\langle 1,0\rangle)\right) \Psi\left(\varphi^{k-2}(\langle\lambda, 1\rangle)\right) \\
\rho \xi_{0} \Psi\left(z\left(\varphi^{k-2}(\langle 1,1\rangle)\langle\lambda, 0\rangle\right) \xi_{0}^{-1}\right. & <\Psi\left(\varphi^{i-1}(\langle 1,0\rangle)\right) \nu
\end{aligned}
$$

where

$$
\begin{aligned}
v & =\Psi\left(\varphi^{k-2}(\langle\lambda, 1\rangle)\right) \Psi\left(\varphi^{k-2}(\langle 1,1\rangle)\langle\lambda, 0\rangle\right) \xi_{0}^{-1} \\
& =\Psi\left(\varphi^{k-1}(\langle\lambda, 1\rangle)\langle\lambda, 0\rangle\right) \xi_{0}^{-1} \\
& =\rho .
\end{aligned}
$$

Therefore

$$
\begin{aligned}
\left.\xi_{0} \Psi\left(z \varphi^{k-2}(\langle 1,1\rangle) c\right)\right) \xi_{0}^{-1} & <\rho^{-1} \Psi\left(\varphi^{i-1}(\langle 1,0\rangle)\right) \rho \\
\xi_{0} \Psi\left(z \varphi^{k-2}(\langle 1,1\rangle) c\right) \xi_{0}^{-1} & <w_{i-1}
\end{aligned}
$$

where the left-hand side is a conjugate of $\Psi\left(\varphi^{i-1}(\langle 1,0\rangle)\right)$ and hence of $w_{i-1}$. This contradicts to the inductive hypothesis that $w_{i-1}$ is a Lyndon word.

Theorem 4.4.1 follows from Lemma 5.4.4, Lemma 5.4.7, Lemma 5.4.8, Lemma 5.4.10 and Lemma 5.4.12.

\section{Concluding remarks}

Out of the four subclasses of the generalized Thue sequences, for three subclasses the canonical Lyndon factors grow proportionally with applications of the substitution $\mu$. In the only case, when $w=1^{k}$, the 
factors are growing with $\mu^{2}$. This fact prevents us from trying to make any kind of conjecture on the general shape of the Lyndon factors for the whole class of generalized sequences of Thue. A study of the Lyndon factorization of further subclasses will be necessary to have a kind of general result. We hope that Definition 3.1 .2 together with Lemma 3.2.4 may be equally useful as they proved to be in the investigation of the four subclasses in the present work.

\section{Acknowledgements}

The author would like to thank the anonymous referees for multiple useful remarks and suggestions towards improving readability of the paper.

\section{References}

[1] J. Berstel, A. de Luca, Sturmian words, Lyndon words and trees, Theoretical Computer Science, 178, (1-2) (1997), 171-203

[2] J. Berstel, M. Pochiolla, Average cost of Duval's algorithm for generating Lyndon words, Theor. Computer Science 132 (1994), 415-425

[3] A. Černý, On a class of infinite words with bounded repetitions, R.A.I.R.O. Informatique théorique/Theoretical Informatics 19, 4 (1985), 337-349

[4] A. Černý, J. Gruska, Modular trellises, In: The Book of L., (Rozenberg., G., and Salomaa, A., eds.), Springer Verlag, Berlin, (1986), 45-60

[5] G. Christol, T. Kamae, M. Mendès-France, G. Rauzy, Suites algébriques, automates et substitutions, Bull. Soc. Math. France 108 (1980), 401-419

[6] A. Cobham, Uniform tag sequences, Math. Syst. Theory 6 (1972), 164-192

[7] G. Duchamp, D. Krob , Lazard's factorizations of free partially commutative monoids, Proc. ICALP (1991), 242-253

[8] J.P. Duval , Factorizing words over an ordered alphabet, Journal of Algorithms 4 (1983), 363-381

[9] A. Ido, G. Melançon, Lyndon factorization of the Thue-Morse word and its relatives, Discrete Mathematics and Theoretical Computer Science, 1, 1 (1997), 43-52

[10] M. Lothaire, Combinatorics on words, Addison-Wesley, Reading, MA. (1983)

[11] G. Melançon, Lyndon factorization of infinite words, Proceedings STACS'96, (C. Puech, R. Reischuk, eds.), LNCS 1046, Springer, Berlin (1996), 147-154

[12] G. Melançon, Lyndon Factorization of Sturmian Words, 8th international Conference on Formal Power Series and Algebraic Combinatorics (FPSAC), Minneapolis (1996). http://dept-info.labri.ubordeaux.fr/ $/$ melancon/RECHERCHE/publications/sturm.ps.gz

[13] G. Melançon, Viennot factorization of infinite words, Information Processing Letters, 60 (1996), 53-57. 
[14] M. E. Prouhet, Mémoire sur quelques relations entre les puissances des nombres, C. R. Acad. Sci. Paris 33 (1851), Cahier 31, 225

[15] P. Séébold, Generalized Thue-Morse sequences, in: Proc. FCT'85, Lecture Notes in Computer Science 199 (1985), Springer-Verlag, , 402-411

[16] P. Séébold, Complément à l'étude des suites de Thue-Morse généralisées, Infromatique théorique et applications 20 (1986), 157-181

[17] R. Siromoney, L. Matthew, V. R. Dare, K.G. Subramanian, Infinite Lyndon words, Inf. Processing Letters 50 (1994), 101-104

[18] A. Thue, Ueber unendliche Zeichenreihen, Videnskabsselskabets Skifter, Math. Naturv. Klasse, Kristiania 7 (1906) 1-22

[19] A. Thue, Über die gegenseitige Lage gleichen Teile gewisser Zeichenreihen, Videnskapsselskapets Skifter, I. Mat. - naturv. Klasse, Kristiania 1 (1912) 1-67

[20] S. Varricchio, Factorizations of free monoids and unavoidable regularities, Theor. Computer Science 73 (1990), 81-89 